\title{
Confined states in large-aspect-ratio thermosolutal convection
}

\section{Alejandro Spina, ${ }^{1}$ Juri Toomre, ${ }^{1}$ and Edgar Knobloch ${ }^{1,2}$ \\ 'JILA, University of Colorado, Boulder, Colorado 80309 \\ ${ }^{2}$ Department of Physics, University of California at Berkeley, Berkeley, California 94720

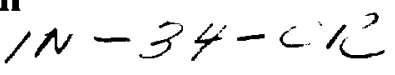

(Received 13 June 1997)

\begin{abstract}
Two-dimensional thermosolutal convection with no-slip boundary conditions is studied using numerical simulations in a periodic domain. The domain is large enough to follow the evolution of phase instabilities of fully nonlinear traveling waves. In the parameter regime studied these instabilities evolve, without loss of phase or hysteresis, into a series of confined states or pulses characterized by locally enhanced heat and solute transport. The wavelength and phase velocity of the traveling rolls within a pulse differ substantially from those in the background. The pulses drift in the same direction as the convection rolls on which they ride but more slowly, and are characterized by an exponential leading front and an oscillatory trailing end. Multiple, apparently stable, states are found for identical parameter values. The qualitative properties of the pulses are in good agreement with the predictions of a third-order phase equation which accounts for the relation between wave number and phase velocity, the oscillatory tails and the multiplicity of states. These properties of the pulses are shown to be a consequence of Shil'nikov dynamics in the spatial domain. [S 106.3-651X(98)04901-0]
\end{abstract}

PACS number(s): 47.20. $-\mathrm{k}, 47.35 .+\mathrm{i}$

\section{THE PHYSICAL PROBLEM}

The dynamics of overstable convection produced by the competition between stabilizing and destabilizing mechanisms in doubly diffusive systems has attracted much interest. Typical of such systems is thermosolutal convection in which this instability usually develops into nonlinear traveling waves. Such waves were studied extensively $[1,2]$ using a combination of bifurcation analysis and numerical simulation in two dimensions, employing periodic boundary conditions with period $2 \pi / k_{r}$ in the horizontal. Here $k_{r}$ is the wave number with which the instability sets in. While such simulations are suitable for the study of the relative stability between traveling and standing waves they exclude longwavelength modes to which such waves can become unstable.

This paper is devoted to the study of long-wavelength instabilities of fully nonlinear traveling waves. For this purpose we employ a relatively large aspect ratio $\Gamma=64$ with periodic boundary conditions applied at $x=0,64$ allowing something of the order of 26-32 roll pairs to form in the spatially uniform state, depending on parameters. We find, and describe in detail, a new dynamical traveling wave state, consisting of one or more nonlinear pulses or confined states, comprising rolls of different wave number, phase velocity, and amplitude than the background wave train, that travel more slowly than the background while transporting more of the heat and solute. We provide convincing evidence that these pulses form as a result of a phase instability of the background wave train, and describe a qualitative theory based on a third-order phase equation that accounts for their structure. In particular, we suggest that both single-pulse and multipulse states are manifestations of Shil'nikov dynamics in the spatial variable and thereby account for the observation, at fixed parameter values, of multiple, numerically stable, pulse states.

Confined states have been the subject of much interest in the last several years, motivated largely by the discovery of such states in binary fluid convection [3]. This system is closely related to thermosolutal convection but differs from it in several crucial ways. In binary fluid convection the stabilizing solute gradient develops in response to the applied adverse temperature gradient; no solute is therefore transported through the system. The traveling waves that are observed appear via a subcritical Hopf bifurcation, and very close to onset are seen to evolve into patches of traveling waves separated by an essentially quiescent fluid. The envelope of these waves moves rather slowly and in some experiments may be stationary. A qualitative theory of this phenomenon, based on envelope equations coupled to the concentration field, appears to account for most of the observed properties of these confined states. It should be noted that the subcriticality of the bifurcation is an essential part of this theory [4], which describes the traveling wave patches in terms of solitary waves coupled to the large scale concentration field [5]. Such waves are in turn to be viewed as homoclinic orbits to the origin in an appropriate amplitude equation, that is, the amplitude increases from zero at $x=-\infty$ to a maximum and then decreases to zero again as $x \rightarrow+\infty$ [6]. Such states have also been found in numerical simulations of the full partial differential equations with experimental parameters and are in excellent agreement with their measured properties [7]

The confined states that form the subject of the present paper are quite different. In particular, they connect a uniform but fully nonlinear wave train at $x=-\infty$ to itself as $x \rightarrow \infty$. Consequently, the traditional amplitude equation approach is inadmissible; the pulses must be described using a real order parameter, the phase of the wave. We are able to locate numerically a regime in which the phase of the wave is apparently conserved, and develop our theory based on phase conservation. As discussed further below, the phase is not always conserved and when it is not our theory no longer applies.

The recent experimental realization of doubly diffusive convection by Predtechensky et al. [8] raises the possibility 
that the pulses described below could be observed in an appropriately focused experiment. In these experiments the destabilizing buoyancy force is produced by an imposed concentration gradient of a second species, instead of an adverse temperature gradient. Consequently the Lewis number (i.e., the ratio of the diffusivities of the stabilizing and destabilizing components) lies typically in the range $0.1-1.0$, a parameter range that forms the focus of the present study. However, in the following we retain the traditional thermosolutal terminology.

This paper is organized as follows. In the next section we describe the system studied and some of its elementary properties. In Sec. III, which forms the bulk of the paper, we describe the results of our numerical simulations. In particular, we discuss the properties of the various fields (temperature, concentration, vorticity) inside the pulses and outside. We provide detailed information on the phase velocity, wave number, and amplitude of the rolls inside and outside a pulse at three different Rayleigh numbers, as well as information about the speed of propagation of the pulses. We also describe the transport properties of the pulses in both vertical and horizontal directions, and compare their transport efficiency with the background wave train. This information forms the basis of the theoretical interpretation of the results advanced in Sec. IV. The paper ends with a brief discussion of the results and an intimation of future work.

\section{THE MATHEMATICAL MODEL}

The nondimensionalized equations describing twodimensional thermosolutal convection can be written in the form [1]

$$
\begin{gathered}
\frac{1}{\sigma}\left[\partial_{t} \nabla^{2} \Psi+J\left(\Psi, \nabla^{2} \Psi\right)\right]=R_{T} \partial_{x} \theta-R_{S} \partial_{x} \Sigma+\nabla^{4} \Psi \\
\partial_{t} \theta+J(\Psi, \theta)=\partial_{x} \Psi+\nabla^{2} \theta \\
\partial_{t} \Sigma+J(\Psi, \Sigma)=\partial_{x} \Psi+\tau \nabla^{2} \Sigma
\end{gathered}
$$

where $\Psi(x, z, t)$ is the stream function, $\Theta(x, z, t)$ and $\Sigma(x, z, t)$ denote departures of the temperature $T$ and concentration $S$ from their linear conduction profiles in the absence of convection, and $J(\Psi, f)$ denotes the Jacobian $\partial(\Psi, f) / \partial(x, z)$. In terms of the stream function the velocity is given by $(u, 0, w)=\left(-\partial_{z} \Psi, 0, \partial_{x} \Psi\right)$. The dimensionless parameters $R_{T}$ and $R_{S}$ are the thermal and solutal Rayleigh numbers, respectively; we shall use the former as our bifurcation parameter. The quantities $\sigma$ and $\tau$ denote the Prandtl and Lewis numbers.

Equations (1)-(3) are to be solved in the domain $(x, z)$ $\in[0, \Gamma) \times[0,1]$, for $t>0$, subject to periodic boundary conditions in the horizontal,

$$
(\Psi, \Theta, \Sigma)(x, z, t)=(\Psi, \Theta, \Sigma)(x+\Gamma, z, t)
$$

and no-slip, fixed temperature and concentration boundary conditions at top and bottom,

$$
u=w=\Theta=\Sigma=0, \quad z=0,1 .
$$

(a)

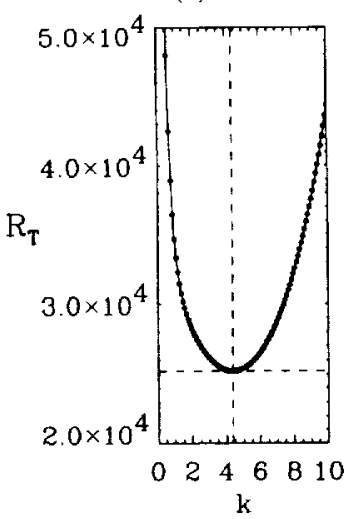

(b)

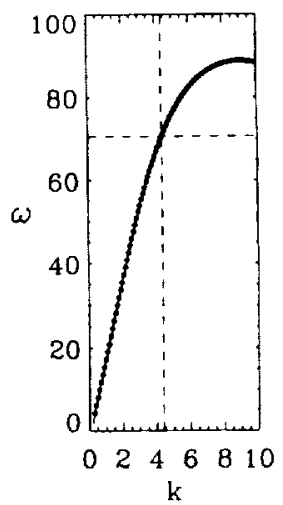

FIG. 1. Neutral stability curves (a) $R_{T}$ vs $k$ and (b) $\omega$ vs $k$ for $\Gamma=64.0, R_{S}=30000.0, \sigma=1.0$, and $\tau=10^{-1 / 2}$. Dots correspond to the discrete modes of the finite domain, which increase in number in proportion to $\Gamma$. The continuous-case critical values provided by linear stability theory are $R_{T}^{(0)}=25251.76, \quad k^{(0)}=4.37, \quad \omega^{(0)}$ $=70.66$.

The velocity boundary conditions translate into the following boundary conditions on the stream function:

$$
\begin{aligned}
& \Psi(x, 0, t)=0, \quad \Psi(x, 1, t)=-m(t), \\
& \partial_{z} \Psi(x, 0, t)=0, \quad \partial_{z} \Psi(x, 1, t)=0,
\end{aligned}
$$

with $m(t)$ determined as part of the solution. The absence of Galilean invariance implies that $m(t)$ is unique; in fact $m(t)$ is the horizontal mass flux resulting from any mean flow $(U(z, t), 0)$ that accompanies the solution:

$$
m(t) \equiv \int_{0}^{1} U(z, t) d z=-\frac{1}{\Gamma} \int_{0}^{1} d z \int_{0}^{\Gamma} d x \frac{\partial \Psi}{\partial z}
$$

For traveling waves $m(t) \neq 0$.

As in earlier studies [1] we use the parameter values $\sigma=1.0, \tau=10^{-1 / 2}$, with $R_{S}=3 \times 10^{4}$ and $\Gamma=64.0$. Figure 1 shows the neutral stability curve $R_{T}$ as a function of the horizontal wave number $k$ and the corresponding frequency $\omega(k)$, both for $\Gamma=64.0$ and $\Gamma=\infty$. The dots indicate the discretized values corresponding to the finite aspect ratio. For these parameter values, the critical (thermal) Rayleigh number, wave number, and oscillation frequency are found to be

$$
\begin{gathered}
R_{T}^{(0)}=25251.76, \\
k^{(0)}=4.37, \\
\omega^{(0)}=70.66 .
\end{gathered}
$$

The large value of $\omega^{(0)}$ indicates that the system is far from the codimension-two point.

With periodic boundary conditions the system (1)-(5) is equivariant under the group $O(2)$ of rotations and reflections of a circle. As a consequence of this symmetry the number of eigenvalues on the imaginary axis is doubled, and two branches of solutions, standing waves (SW) and traveling 
waves (TW), bifurcate simultaneously from the conduction solution. Specifically, near onset,

$$
\begin{aligned}
\Psi(x, z, t)= & \operatorname{Re}\left[A_{L}(t) e^{i\left[k x+\phi_{L}(t)\right]}+A_{R}(t) e^{i\left[k x-\phi_{R}(t)\right]}\right] f(z) \\
& +O(2)
\end{aligned}
$$

where $A_{L}(t)$ and $A_{R}(t)$ are the (real) amplitudes of left- and right-traveling waves, satisfying the equations

$$
\begin{aligned}
& \dot{A}_{R}=\left(\lambda+a A_{L}^{2}+b A^{2}+\cdots\right) A_{R}, \\
& \dot{A}_{L}=\left(\lambda+a A_{R}^{2}+b A^{2}+\cdots\right) A_{L} .
\end{aligned}
$$

Here $A^{2} \equiv A_{R}^{2}+A_{L}^{2}$ is the square of the total amplitude, and $\lambda \pm i \Omega$ are the (double) eigenvalues of the linear stability problem. Generically, these equations possess only three persistent solutions: the conduction state $\left(A_{L}, A_{R}\right)=(0,0)$, lefttraveling waves $(A, 0)$, and standing waves $(A / \sqrt{2}, A / \sqrt{2})$; all other solutions can be obtained using translations and/or reflections of these elementary solutions. The stability of these solutions is determined by the real coefficients $a, b$ as described by Knobloch [9]. With stress-free boundary conditions at top and bottom $b \equiv 0[1,9]$; this is not so, however, for the more realistic no-slip boundary conditions (5). Renardy [10] shows that for the parameters employed both TW and SW bifurcate subcritically at onset. Thus neither branch is stable near onset. The large-amplitude traveling waves we find numerically at supercritical Rayleigh numbers probably acquire stability at finite amplitude via a saddle-node bifurcation followed by the shedding of a branch of modulated traveling waves, as described in Ref. [11].

Equations (1) - (3) with boundary conditions (4) - (6) were solved using a hybrid finite-difference and spectral scheme. Spectral decomposition in the horizontal with 16 grid points per unit length provided 512 Fourier modes for the spectral decomposition at each $z$ level of the grid. A 33 point equallength finite-difference grid in the vertical provided an economic compromise between the resolution needs of the boundary layers and those of the long-range fluxes. We worked with the equations of motion in their biharmonic form (1)-(3) rather than the more traditional streamfunction-vorticity formulation in order to avoid (a) inversion of the Poisson operator, and (b) difficulties arising from the coupling between the stream function and vorticity [12]. These are associated with the peculiar nature of the boundary conditions for the stream function $\Psi$ and the initial conditions on the vorticity $\omega$, which lacks boundary conditions [13]. In the biharmonic formulation the specification of both Dirichlet and Neumann boundary conditions for $\Psi$ poses no difficulty because both are required to supplement the fourthorder elliptic operator $\nabla^{4}$ present in Eq. (1).

Solutions were first computed with fictitious boundary conditions for the stream function $\Psi$, namely, $\Psi=\partial_{z} \Psi=0$, at $z=0,1$. This allows for larger time steps in the numerical code and a fast approach to a stationary solution. These boundary conditions cancel the overall lateral fluid transport, but the dynamics that result from them are close to the true ones when the net horizontal transports are small. One simulation was run from random initial conditions until the solution matured, and then solutions for other values of $R_{T}$ were found by perturbing the previous ones. Once stationarity was achieved in this manner, the resulting fields were used as starting points for subsequent simulations in which the real boundary conditions for the stream function were used $\left[\partial_{z} \Psi=0\right.$, at $z=0,1$, with $\left.\Psi(x, 0, t)=0, \Psi(x, 1, t)=-m\right]$. Other solutions were run fully from random initial data for checking purposes.

\section{RESULTS}

The nonlinear simulations reported below explore the Rayleigh number range from $R_{T}=1.15 R_{T}^{(o)}$ to $1.55 R_{T}^{(o)}$ and cover the regime from just prior to pulse formation to the regime where phase is no longer conserved and solutions appear weakly chaotic. We concentrate on the range characterized by phase conservation, and present detailed results for three values of $R_{T}$, viz, $1.19 R_{T}^{(o)}, 1.23 R_{T}^{(o)}$, and $1.27 R_{T}^{(o)}$.

The efficiency of convection is measured by the thermal and solutal Nusselt numbers. We define these dimensionless numbers as the ratios of the time-averaged vertical heat or solute flux to the corresponding conductive flux in the absence of convection through a given plane $z=$ const. Specifically, we define the thermal and solutal Nusselt number densities $N_{T}(x, z)$ and $N_{S}(x, z)$ in dimensionless variables to be

$$
N_{T}(x, z)=\left\langle-\frac{\partial T(x, z, t)}{\partial z}+w(x, z, t) T(x, z, t)\right)
$$

and

$$
N_{S}(x, z)=\left\langle-\frac{\partial S(x, z, t)}{\partial z}+\tau^{-1} w(x, z, t) S(x, z, t)\right\rangle .
$$

where \langle\rangle indicates the time average. The thermal Nusselt number $N_{T}(x)$ is then given by

$$
N_{T}(x)=\int_{0}^{1} N_{T}(x, z) d z \text {. }
$$

In the simulations reported here no qualitative difference was found between the values of the vertically averaged Nusselt number $N_{T}(x)$ and those of $N_{T}(x, z)$ evaluated at midlayer. The total thermal Nusselt number is usually defined as

$$
N_{T}=\frac{1}{\Gamma} \int_{0}^{\Gamma} N_{T}(x) d x
$$

We shall find it convenient to also introduce reduced thermal Nusselt numbers, defined by

$$
N_{T, \Delta}=\left(\frac{1}{\int_{\Delta} d x}\right) \int_{\Delta} N_{T}(x) d x .
$$

These measure the transport properties of heat in subdomains $\Delta$ of the overall domain of extent $\Gamma$. Solutal Nusselt numbers $N_{S}(x), N_{S}$, and $N_{S, \Delta}$ are similarly defined.

For a steadily traveling pattern with or without a pulse (but no phase slips) all global quantities are time independent. This is not so, however, for the reduced Nusselt numbers which are modulated because of the passage of rolls into and out of the pulse. In addition to the above quantities the 
local kinetic energy density $E(x, z, t)=\frac{1}{2} \mathbf{u} \cdot \mathbf{u}$, and the enstrophy density $Z(x, z, t)=|\omega|^{2}$ are used as diagnostics, with $E$ and $Z$ being the values of the associated integrals over the full domain. Here $\boldsymbol{\omega}=\nabla \times \mathbf{u}$ is the vorticity.

Lateral and vertical fluxes of heat and solute have been computed, as have the mean lateral velocity and mass flux. When the fluid motion attains a statistically stationary regime these quantities become time independent. The wavelength of the convective rolls both inside and outside the pulses is an important diagnostic of the phase and has been carefully measured. The propagation speeds of the rolls inside and outside the pulses were computed by tracking zeros of the stream function $\Psi$ at midlayer. The presence of a "mirror-glide" symmetry [2,14-16] makes the midline the natural place for these measurements. Finally, moving averages of several of the fields have been obtained. Such averages are computed in reference frames moving at the pulse velocity. This procedure filters out small-scale structures, revealing the pattern of large-scale behavior.

\section{A. Structure of the fields}

At the lower end of our Rayleigh number regime random perturbations of the conduction state $\Psi=\theta=\Sigma=0$ evolve into a spatially uniform traveling wave train; left- and righttraveling wave trains are equally likely. Although of modest amplitude these solutions reveal that both $\Theta$ and $\Sigma$ have a trapezoidal, highly nonharmonic shape that cannot be described quantitatively by a weakly nonlinear expansion near onset $[15,16]$. In these states all the fields have the "mirrorglide" symmetry

$$
f(x, z, t)=-f\left(x+\frac{\lambda}{2}, 1-z, t\right),
$$

which is a combination of a translation by half a wavelength in the $x$ direction with a mirror reflection through the $z=\frac{1}{2}$ plane $[2,14-16]$. This symmetry persists even at large Rayleigh numbers as long as the number of roll pairs is conserved during their time evolution. Associated with these states small-amplitude long-wavelength modulations were sometimes observed; these are believed to be long-lived transients.

As the Rayleigh number $R_{T}$ is increased propagating pulses on a background of traveling rolls appear. When the simulations start from a random perturbation of the conduction state at these values of $R_{T}$ there appear several such domains with different wavelengths and with varying widths and propagation velocities. During an initial transient regime these domains move and recombine until they attain their ultimate number. When two such pulses remain after this first stage, their evolution continues until they reach a final equilibrium separation that is preserved (with slight modulations in time) as they move, both at the same speed, in the direction of the background rolls. Simulations carried out with Rayleigh numbers at $1.31 R_{T}^{(o)}$ or above produced states that failed to settle down into a stable coexistence of the different domains.

We now define the quantities that will be used to report our findings and describe how they were measured. For this purpose we split the domain into the pulse and the back- ground, indicated by subscripts $p$ and $b$, respectively, such that $\Delta_{p}+\Delta_{b}=\Gamma$. The boundary separating these regions is defined in terms of the wavelength as measured from $\Psi(x, z, t)$ at $z=\frac{1}{2}$; the pulse region is defined as the region with less than average wavelength, with the background region forming the complement.

Roll wavelength $\lambda_{r}(x)$ : The length of each roll was measured at midlayer and used to define the local semiwavelength. A discrete function was thus built whose ordinates are the midpoint coordinates of each roll and whose abscissas are twice the measured length of the corresponding roll. The roll wavelength $\lambda_{r}(x)$ was obtained by fitting a continuous curve through these points. The average roll wavelength $\bar{\lambda}_{r}$ was computed from the continuous fit using the expression

$$
\bar{\lambda}_{r}=\frac{1}{\Gamma} \int_{0}^{\Gamma} \lambda_{r}(x) d x
$$

The roll wavelength in a pulse was computed using

$$
\bar{\lambda}_{r, p}=\frac{1}{\delta_{p}} \int_{\delta_{p}} \lambda_{r}(x) d x,
$$

where the subportion $\delta_{p}$ of the pulse region $\Delta_{p}$ involves rolls with nearly uniform wavelengths, namely, with wavelengths within $20 \%$ of the relative minimum value in the pulse (cf. Fig. 10). The wavelength in the background was obtained from

$$
\bar{\lambda}_{r, b}=\frac{1}{\Delta_{b}} \int_{\Delta_{b}} \lambda_{r}(x) d x
$$

where $\Delta_{b}$ is the background region of the traveling pattern.

Roll phase velocity $v_{r}(x)$ : A discretized function was computed by measuring the phase velocity of the points at midlayer where the stream function vanishes. A continuous interpolation of the sampled values yields $v_{r}(x)$. The average roll velocity $\bar{v}_{r}$, and the roll velocity within the pulse $\bar{v}_{r, p}$ and in the background $\bar{v}_{r, b}$ were then computed in an analogous way to their wavelength counterparts. These quantities are distinct from the pulse velocity $v_{p}$.

$$
\text { 1. } R_{T}=1.19 R_{T}^{(o)}
$$

Figure 2(a) shows a space-time plot of a one-pulse state at $R_{T}=1.19 R_{T}^{(o)}$. Only clockwise streamlines are shown. The pulse travels in the same direction as the background traveling rolls, but at a smaller speed: the rolls travel with an average speed of $\bar{v}_{r} \approx 14.21$, while the pulse moves at $v_{p}$ $\approx 5.88$. The complete solution consists of 26 pairs of convecting rolls with an average wavelength $\bar{\lambda}_{r} \approx 2.12$, but the local wavelength inside the pulse $\left(\bar{\lambda}_{r, p} \approx 1.31\right)$ is seen to be approximately one-half the wavelength in the background $\left(\bar{\lambda}_{r, b} \approx 2.86\right)$. These wavelengths are almost constant, except in the vicinity of the interface between the pulse and the background. The presence of the pulse thus indicates stable coexistence of regions with rolls of different amplitudes, frequencies, and wave numbers. The pulse is a moving region that the rolls have to traverse. In order to do so they shrink laterally, reduce their overall horizontal speed, and spin more 


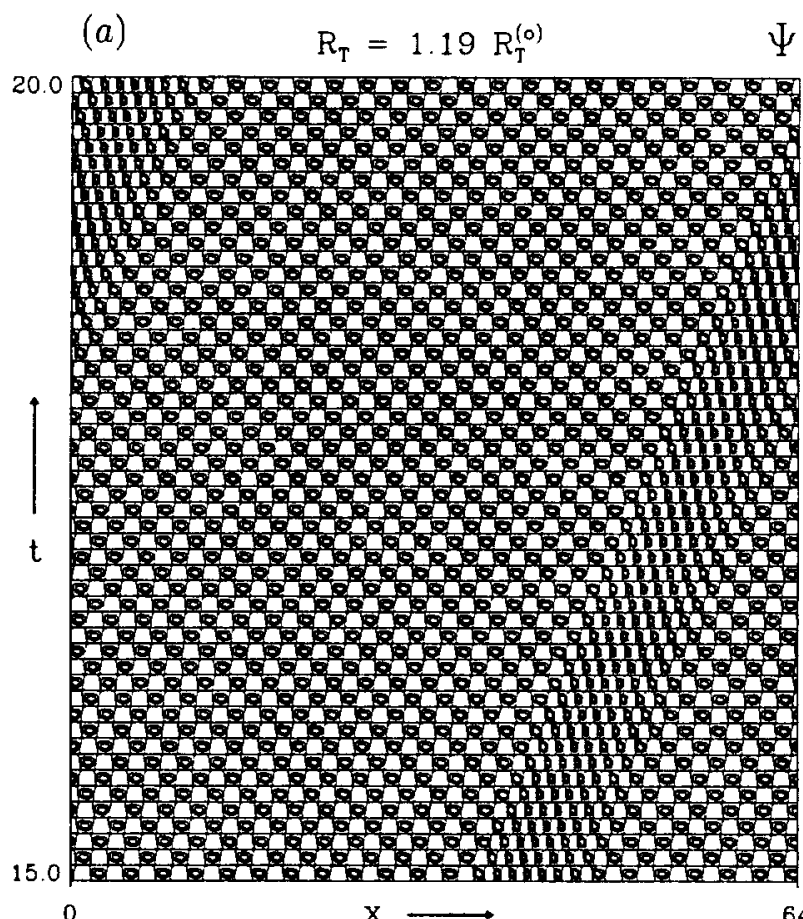

$\Psi$

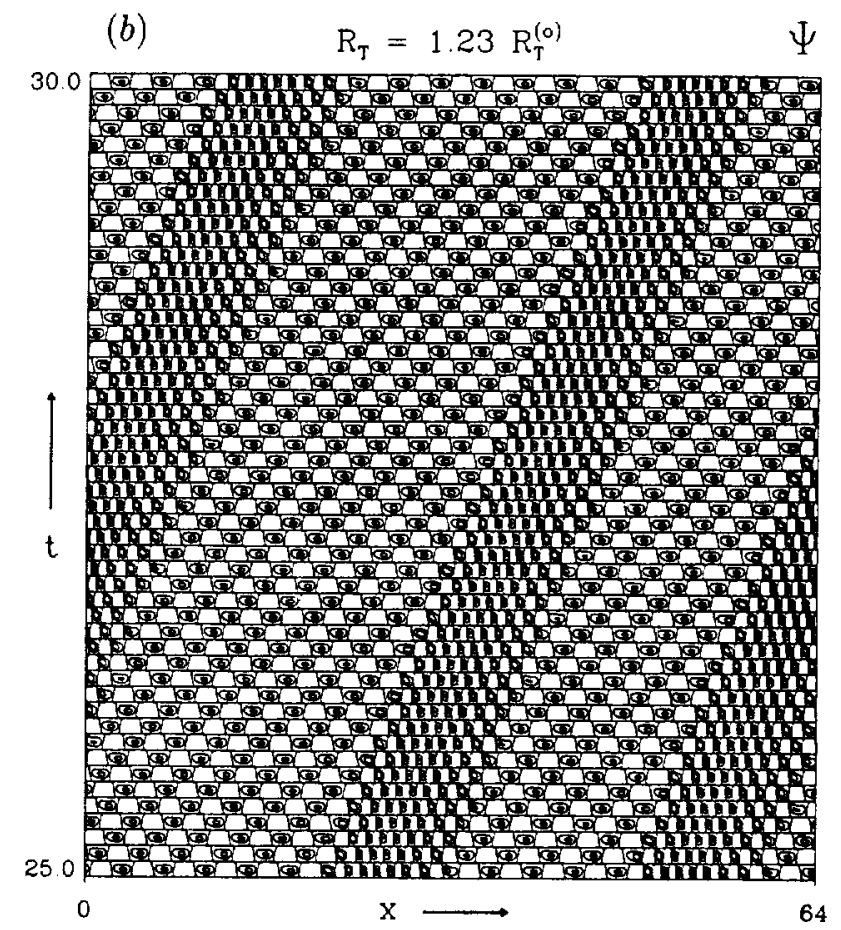

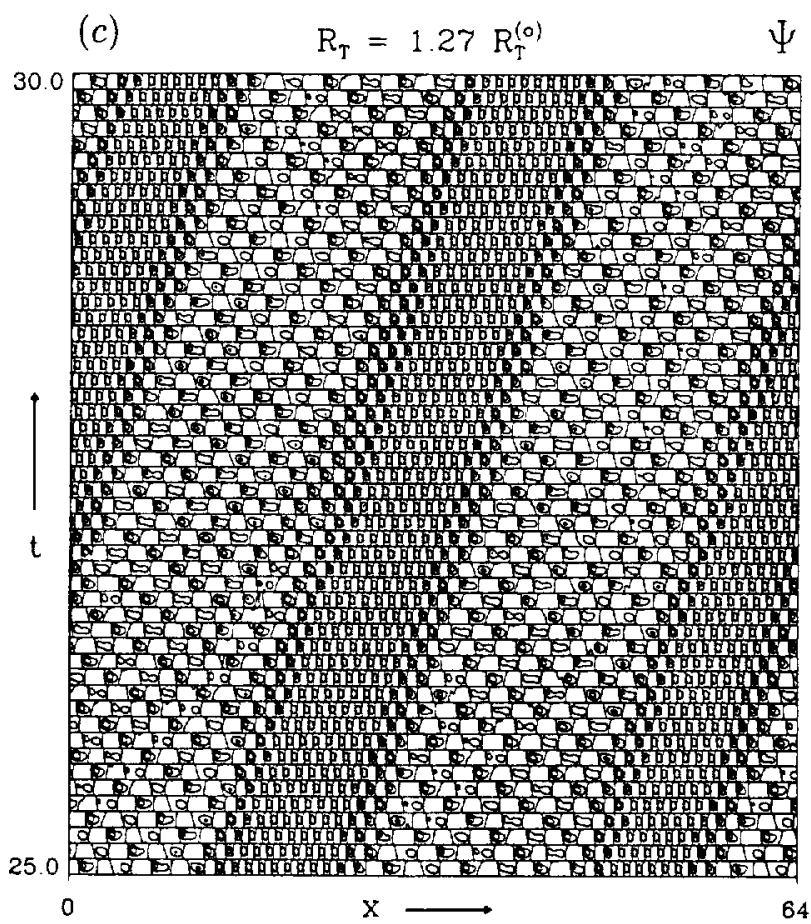

FIG. 2. Traveling waves and pulses in a sequence of equally spaced snapshots of the stream function $\Psi(x, z, t)$ in the full computational domain, with time increasing upward. Rolls and pulses both propagate rightward. (a) One-pulse state for $R_{T}=1.19 R_{7}^{(0)}$ involving 26 roll pairs, $\bar{\lambda}_{r} \approx 2.12, \bar{\lambda}_{r, b} \approx 2.86, \bar{\lambda}_{r, p} \approx 1.31, \bar{v}_{r} \approx 14.21, \bar{v}_{r . h} \approx 15.01, \bar{v}_{r, p} \approx 10.05, v_{p} \approx 5.88$. (b) Two-pulse state for $R_{T}=1.23 R_{T}^{(0)}$ involving 26 roll pairs, $\bar{\lambda}_{r} \approx 2.38, \bar{\lambda}_{r . b} \approx 3.68, \bar{\lambda}_{r, p} \approx 1.21, \bar{v}_{r} \approx 13.11, \bar{v}_{r, b} \approx 15.05, \bar{v}_{r, p} \approx 8.57, v_{p} \approx 5.42$. (c) Two-pulse state for $R_{T}=1.27 R_{T}^{(0)}$ involving 32 roll pairs, $\bar{\lambda}_{r} \approx 2.45, \bar{\lambda}_{r . h} \approx 3.43, \bar{\lambda}_{r, p} \approx 1.11, \bar{v}_{r} \approx 10.66, \bar{v}_{r, b} \approx 12.83, \bar{v}_{r, p} \approx 7.24, v_{p} \approx 4.49$.

vigorously until they pass out of the pulse region. For comparison, at $R_{T}=1.15 R_{T}^{(o)}$ (not shown) the solution also consists of 26 roll pairs but the pulse is absent and the wavelength uniform. Careful numerical investigation indicates that the pulse appears once $R_{T}$ exceeds a well-defined threshold and that the formation process is nonhysteretic.
Figure 3(a) shows a closeup view of the fields. The temperature contours are smoother than the solute ones; due to its small diffusivity, solute is transported predominantly by fluid advection with very little diffusion, while the high diffusivity of the temperature smoothes out its contours more effectively. Plume structures can be seen to form inside the 

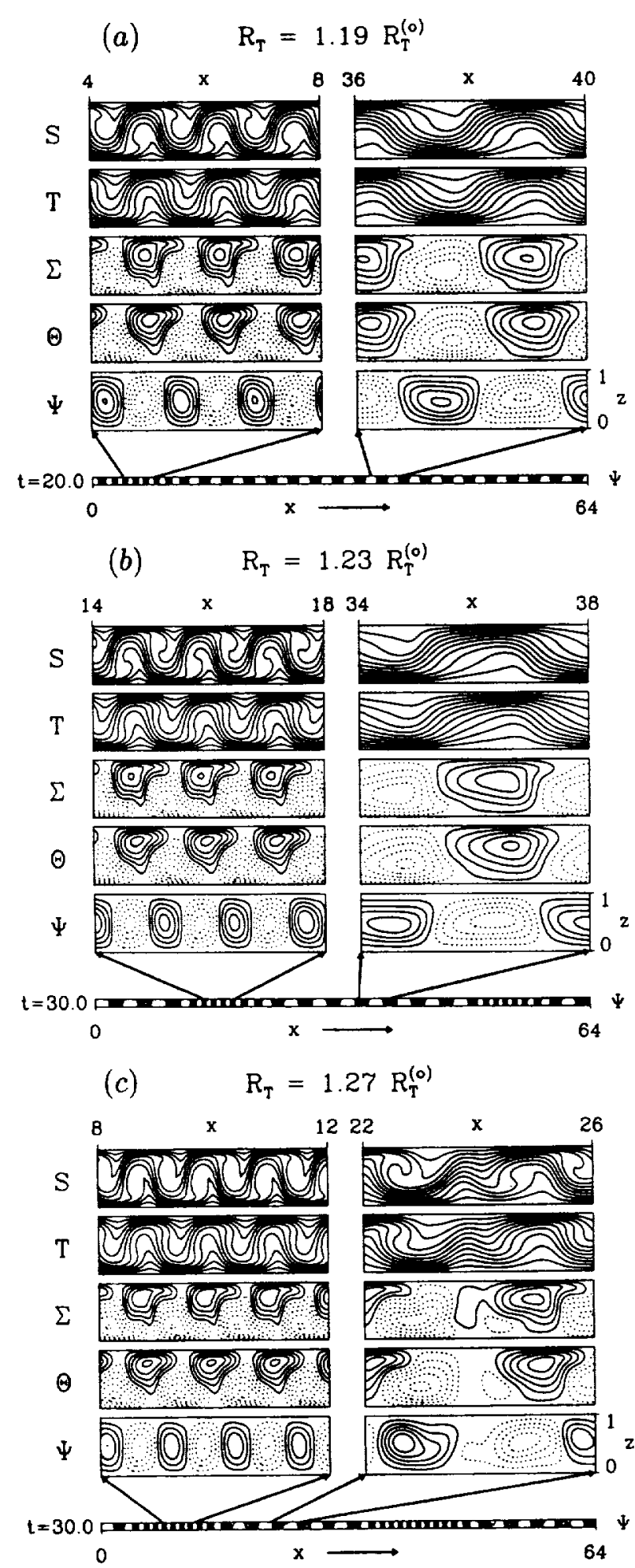

FIG. 3. Closeup views of the field structures inside the pulse region (left panels) and in the background (right panels) at a particular instant in time, showing (from bottom to top) the stream function $\Psi$, the temperature perturbation $\theta$, the concentration perturbation $\Sigma$, the temperature $T$, and the concentration $S$. (a) $R_{T}$ $=1.19 R_{T}^{(o)}$. (b) $R_{T}=1.23 R_{T}^{(o)}$. (c) $R_{T}=1.27 R_{T}^{(o)}$.

pulse with a characteristic mushroom shape caused by the lateral bending of the upward plumes of heat and solute. Although the stream function in Fig. 3(a) has nearly the same number of contour lines inside and outside the pulse the contour lines inside the pulse are more tightly packed, indicating increased gradients, particularly in the horizontal, and hence increased vertical velocities. Stronger plumes will thus be found within the pulse, a fact corroborated by the larger kinetic energy densities found there (not shown). Both ascending and descending plumes tilt leftward in such a righttraveling wave. In contrast to $R_{T}=1.15 R_{T}^{(o)}$ (not shown) the solute contours possess clear regions of localized gradient inversion inside the pulses, indicating a local destabilization by the solutal field. The temperature also reveals regions of localized gradient inversion, but mild in comparison to those of the solute. No such regions of inverted gradients are present outside the pulse at this value of the Rayleigh number. Outside the pulse kinetic energy is mostly dissipated in boundary layers near the top and bottom, where the no-slip boundary conditions force the velocity to vanish, while in the pulse region more kinetic energy is dissipated within the bulk of the fluid, hinting at a more active participation of internal friction mechanisms between the counterpropagating upward and downward plumes of fluid.

Figure 4(a) shows the Nusselt number inside and outside the pulse. The values on the right correspond to the averaged Nusselt number $N_{T}$ and to the reduced Nusselt numbers inside the pulse $\left(N_{T, p}\right)$ and in the background $\left(N_{T, b}\right)$. The Nusselt number $N_{T}(x)$ oscillates vigorously inside the pulse. When averaging these curves to obtain the reduced Nusselt number, a larger value is obtained inside the pulse region than outside, indicating that more efficient transport of heat takes place inside the pulse. Up to an overall scale the solutal Nusselt number $N_{S}(x)$ behaves in the same way and is omitted. Thus although the region occupied by the pulse is $19 \%$ of the total length of the convection domain, $24 \%$ of the total heat transport and $26 \%$ of the total solute transport is accomplished within the pulse.

$$
\text { 2. } R_{T}=1.23 R_{T}^{(o)}
$$

As the Rayleigh number is increased, more pulses are seen in mature states. Figure 2(b) shows a state with two right-traveling pulses at $R_{T}=1.23 R_{T}^{(o)}$. The number of roll pairs in the pattern remains at 26 , the same as in the onepulse case. The overall portion of the physical domain occupied by the pulses is now $36 \%$, and the local wavelength of the rolls inside the pulses $\left(\bar{\lambda}_{r, p} \approx 1.21\right)$ is roughly $33 \%$ of that of the rolls outside the pulses $\left(\bar{\lambda}_{r, b} \approx 3.68\right)$. The rolls move to the right at an average velocity of $\bar{v}_{r} \approx 13.11$, and both pulses travel to the right with the same velocity $v_{p} \approx 5.42$, approximately $41 \%$ of the average velocity of the rolls. Although both pulses are of roughly the same length and resemble the single pulse of the previous case, they are not equally spaced. The distance between the two pulses seems to be an equilibrium separation that is unaffected by small perturbations.

The corresponding fields and Nusselt numbers are shown in Figs. 3(b) and 4(b). The two pulses now occupy 36\% of the domain and carry $47 \%$ of the heat transport and $50 \%$ of the solute transport. The pulses are characterized by large regions of localized gradient inversion in the solutal field and milder ones in the temperature field. No such gradient inversion is observed outside them. 

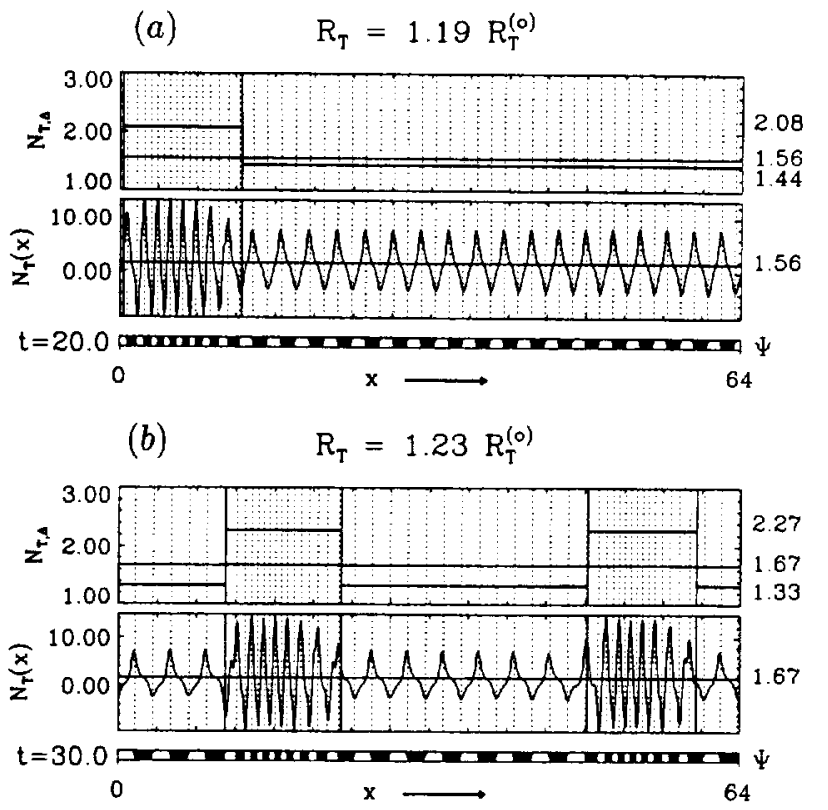

(c)

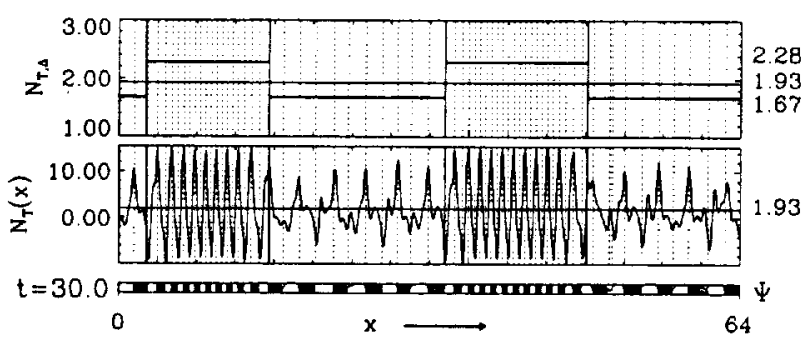

FIG. 4. Thermal Nusselt numbers at a particular instant in time. Vertical lines indicate roll boundaries (dotted), and pulse boundaries (solid). The bottom panel shows the pointwise behavior of $N_{T}(x)$ oscillating around the total thermal Nusselt number $N_{T}$ indicated at the right. In the top panel the numbers on the right indicate the total Nusselt number (middle), and the reduced Nusselt numbers inside (top) and outside (bottom) the pulse region. Solutal Nusselt numbers behave in a qualitatively similar way (see Table I for a summary). (a) $R_{T}=1.19 R_{T}^{(o)}$. (b) $R_{T}=1.23 R_{T}^{(0)}$. (c) $R_{T}$ $=1.27 R_{T}^{(o)}$.

Figure 5 shows the instantaneous profiles of the mean lateral velocity $\bar{u}(z, t)$ and lateral heat flux density $\overline{Q_{T}}(z, t)$ at $R_{T}=1.23 R_{T}^{(o)}$, with overbars denoting horizontal averages over either the whole domain or over the pulse and background regions. The profiles of the lateral solute flux density $\overline{Q_{S}}(z, t)$ are shown as dashed lines in the second set of panels. The left column contains averages computed along the whole physical domain, the middle column shows averages computed within the pulse, and the right column those computed in the background region ahead of the pulse. The contour plot of the stream function below the two panels identifies the regions used in the center and right column averages. The mean lateral velocity $\bar{u}$ changes direction three times in the vertical, but the overall mass flux $m$ is directed toward the left.

Figure 6 shows the local phase velocity as a function of position in the domain in the form of a space-time plot. The pulses are associated with localized deficits in the phase velocity. Like the wavelength the phase velocity is nearly

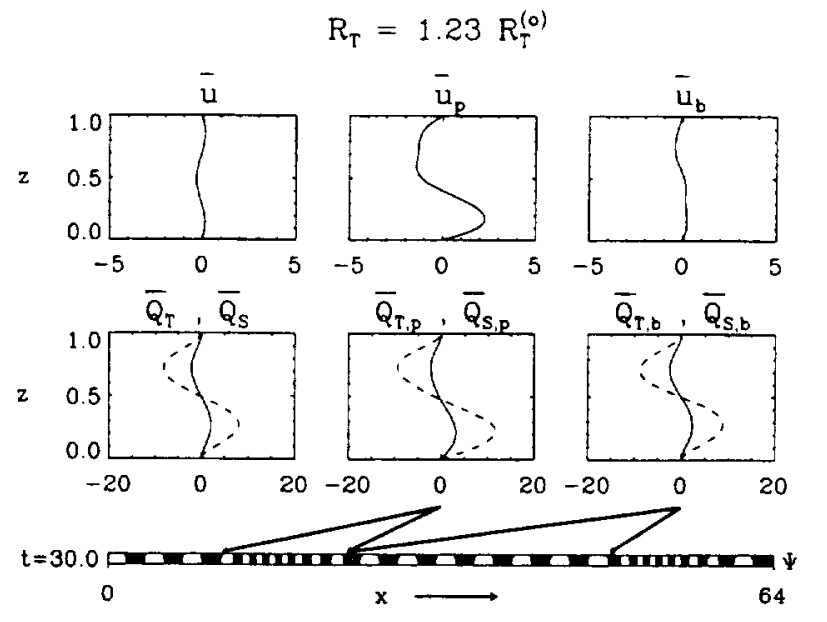

FIG. 5. Instantaneous vertical profiles of mean lateral velocity (top panels) and lateral heat flux density (bottom panels, continuous lines) and lateral solute flux density (bottom panels, dotted lines) for $R_{T}=1.23 R_{T}^{(0)}$. Left panels correspond to horizontal averages performed over the whole domain. Center (right) panels depict these same averages performed only over the pulse (background) region, as indicated by the reference panel at the bottom.

piecewise constant, with relatively sharp transitions near the pulse boundaries. The rapid albeit smooth transition between them produces a clear resolution of the pulse boundaries when the time slices are taken together as in Fig. 6. This is so for the roll wavelengths as well [see Fig. 10(b)]. The wave-

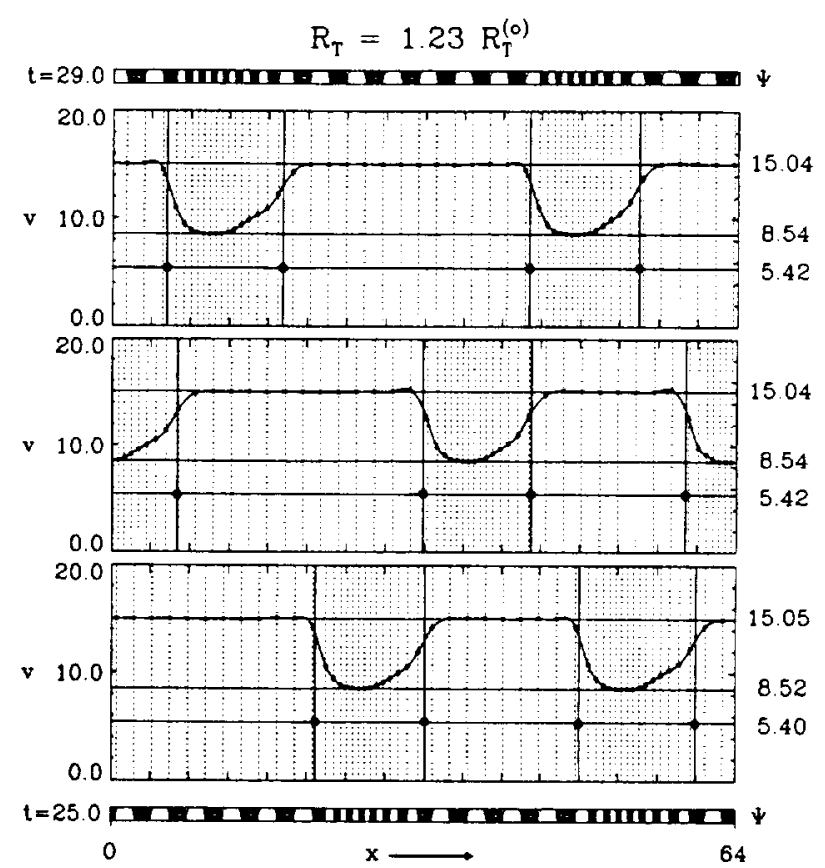

FIG. 6. The roll phase velocity $v_{r}$ as a function of position in a two-pulse state at $R_{T}=1.23 R_{T}^{(0)}$ with 26 roll pairs at three times $t=25,27,29$. Small solid dots indicate computed values. Vertical lines indicate roll boundaries (dotted), and pulse boundaries (solid). The reference stream function panels correspond to the first (bottom) and last (top) phase velocity plots. Values at the right side of each panel are (from top to bottom) the average roll velocity in the background, the roll velocity at the center of the pulse, and the pulse velocity. 
lengths inside the pulses and outside are nearly constant, and both pulses show a clear difference between their leading and trailing ends. The presence of the second pulse is necessarily responsible for the larger wavelength of the rolls outside the pulse region. Associated with the trailing interfaces one can now discern small superposed oscillations in both the local wavelength and phase velocity [cf. Fig. 10(b)]. The asymmetry between the leading and trailing ends of the pulses is present in all diagnostic quantities and is characteristic of all the pulses discussed here; it provides the key to the theory discussed in Sec. IV.

$$
\text { 3. } R_{T}=1.27 R_{T}^{(o)}
$$

Figure 2(c) also shows two rightward moving pulses, this time for $R_{T}=1.27 R_{T}^{(0)}$. In this case 32 pairs of convecting rolls are present with an average wavelength of $\bar{\lambda}_{r} \approx 2.45$. Although this state was obtained from the previous one ( $R_{T}=1.23 R_{T}^{(o)}$ ) by slowly increasing the thermal driving force, the total number of rolls in the pattern has changed. Therefore this state of the fluid motion is not a small perturbation of the previous one. The local wavelength of the rolls inside the pulses $\left(\bar{\lambda}_{r, p} \approx 1.11\right)$ is still roughly $32 \%$ of that outside them $\left(\bar{\lambda}_{r, b} \approx 3.43\right)$, but the pulse spacing is now uniform. The average velocity of the rolls is $\bar{v}_{r} \approx 10.66$, and that of the pulses is $v_{p} \approx 4.49$, approximately $42 \%$ of the average velocity of the rolls.

The shape of the rolls in the central part of the pulses remains quite homogeneous, but the contour lines outside the pulses appear more disordered, suggestive of lateral erosion at the boundaries of the pulses where the stream function now attains larger values than inside. The trailing pulse is slightly larger than the leading one but the roll wavelengths away from the interfaces remain constant and similar in both pulses. As in the previous case, the relative pulse separation remains constant once a stationary regime is achieved.

Figures $3(\mathrm{c})$ and $4(\mathrm{c})$ show that the trends observed at $R_{T}=1.19 R_{T}^{(o)}$ and $R_{T}=1.23 R_{T}^{(o)}$ are enhanced. The pulses are still characterized by strong convection and solute gradient inversion, but now regions of local gradient inversion in the solutal field can also be found outside the pulses. The tight packing of the convection rolls inside the pulses makes these gradient inversion regions less extended inside the pulses than outside, where the bent plumes can be ejected horizontally for larger distances, as can be seen in the solutal field $S$ in Fig. 3(c). Inside the pulses, on the other hand, they are forced to recirculate and the gradient inversion is weaker. The stream function contours inside the pulses retain their symmetry; outside the pulses the maximum of the stream function is displaced laterally from the geometric center of the rolls, creating large horizontal gradients in $\Psi$ and therefore large vertical velocities characteristic of strong ascending and descending plumes of fluid. This accounts for the regions of gradient inversion in the solute outside the pulses, and is reflected in larger kinetic energy density there. The two pulses occupy $44 \%$ of the domain and transport $54 \%$ of the heat and $57 \%$ of the solute.

$$
\text { 4. } R_{T}=1.55 R_{T}^{(o)}
$$

For even higher values of the Rayleigh number pulses of constant form are no longer present. Figure 7 shows a trav-

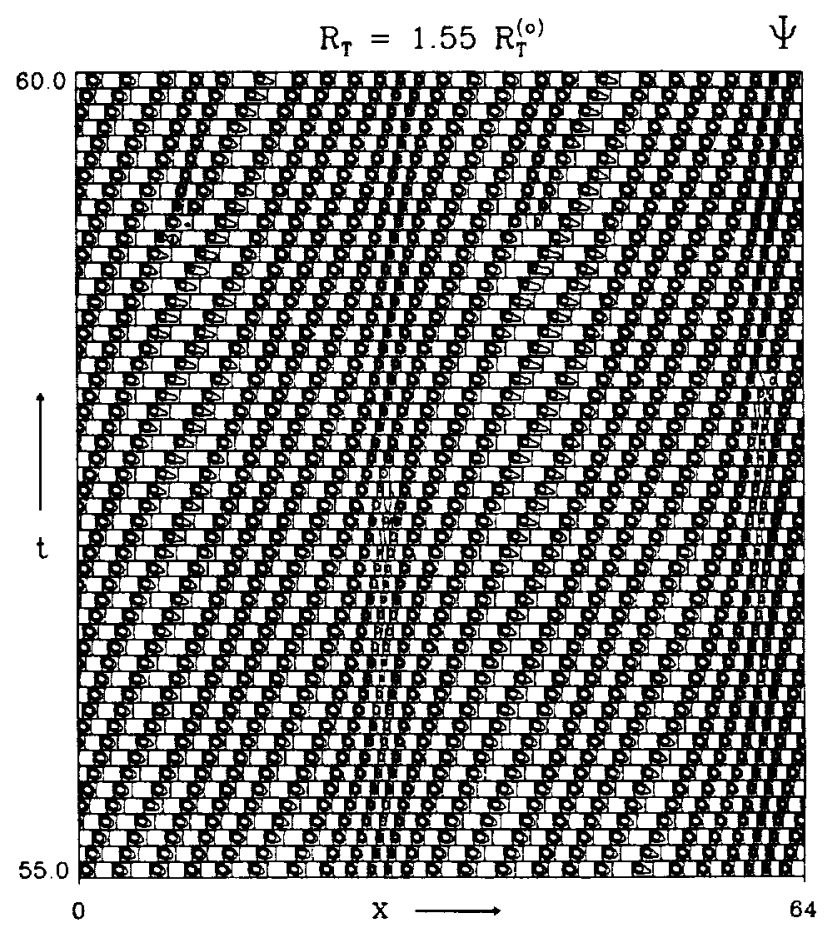

FIG. 7. As for Fig. 2, but showing a traveling wave at $R_{T}$ $=1.55 R_{T}^{(0)}$. Two nearly stationary pulses are present. Spatiotemporal defects form both inside the pulses and in the background; phase is no longer conserved.

eling wave pattern at $R_{T}=1.55 R_{T}^{(o)}$. The pulses are replaced by a couple of almost stationary regions of very small horizontal extent that the pulses have to traverse. These "barriers" serve as phase sinks: two events leading to the disappearance of a roll pair can be discerned, one in each "barrier." In contrast the background region serves as a phase source: two events creating an additional roll pair can be seen in the time interval shown. It appears that the number of roll pairs remains statistically stationary, with the number of roll-splitting events in the background balanced by roll-annihilation events in the slow-moving "barriers." The presence of these spatiotemporal defects in the pattern indicates that phase is no longer conserved.

\section{Mutated states}

We have found that it is possible to generate multiple, numerically stable states for fixed parameter values by a process we call mutation. In this process selected parts of a stable wave train were compressed while others were expanded, with the overall number of roll pairs kept unchanged. Such an initial condition obtained by "gluing" the pieces back together was then evolved by time stepping forward in time. If it was the pulse that was compressed the number of rolls in the pulse decreased rapidly as the system attempted to recover its equilibrium pulse wavelength. In contrast, when the background rolls were compressed a new pulse would often develop, again on a fast time scale. In all cases the resulting state was evolved until a numerically stable final state was reached. In this way different stable states could be manufactured for the same parameter values. The new states would typically differ from the original state in the overall roll number, the separation of the pulses, and in 
(a)

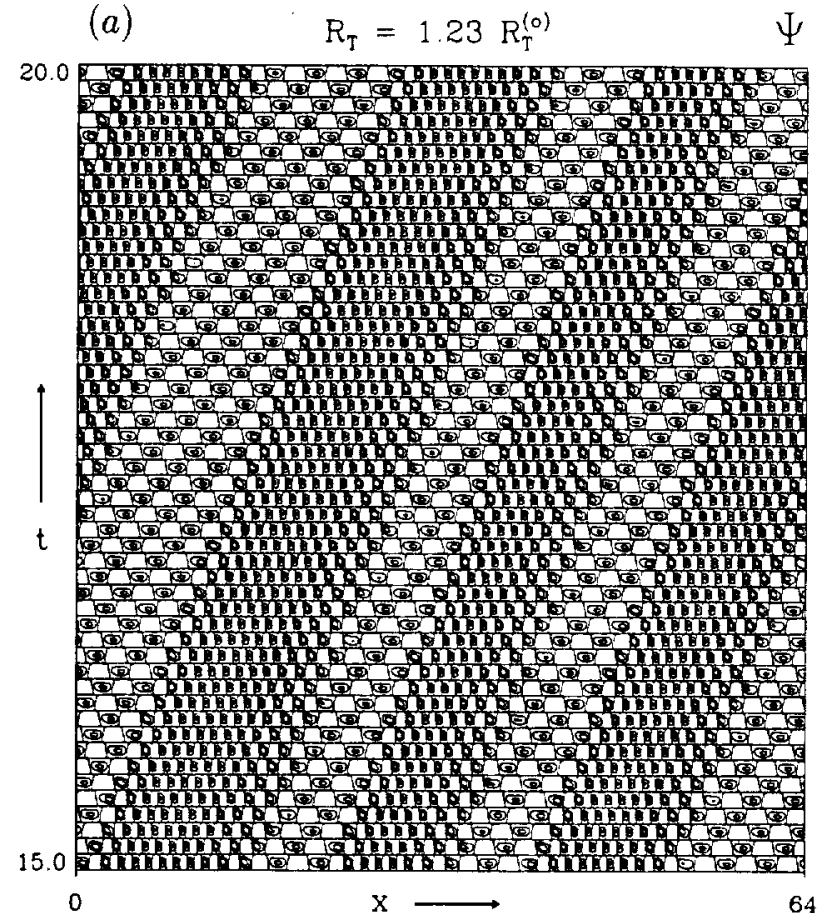

(b)

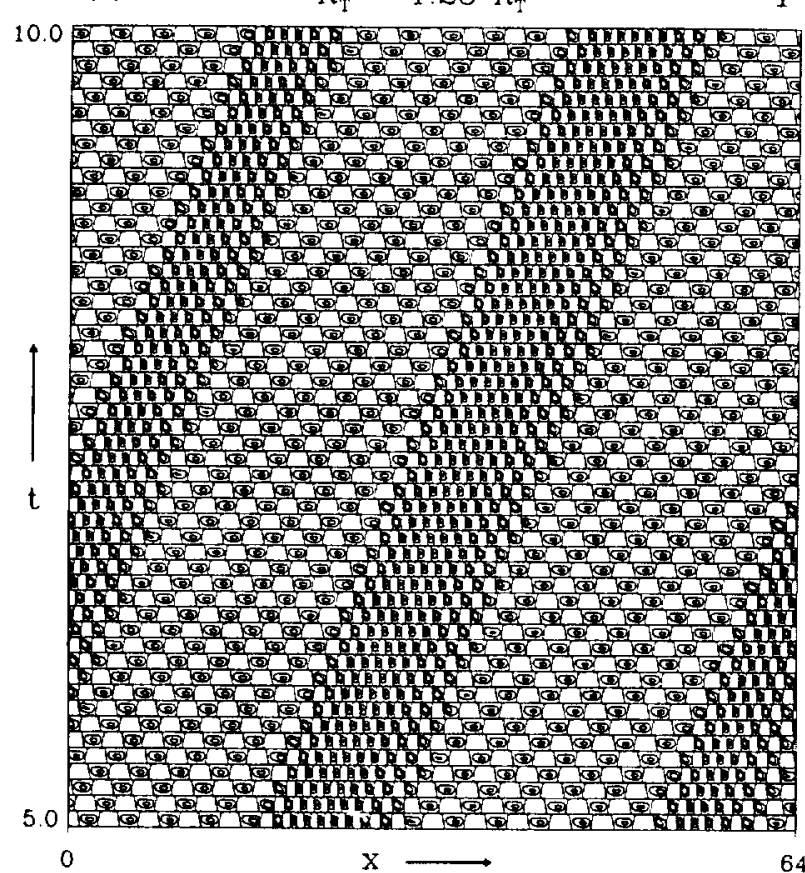

FIG. 8. As for Fig. 2, but showing for $R_{T}=1.23 R_{T}^{(t)}$ (a) a threepulse state, and (b) a two-pulse state, both obtained by mutation of the two-pulse state shown in Fig. 2(b) for the same $R_{T}$. The twopulse state differs from the 26 roll pair state shown there, although it has the same number of rolts.

the number of oscillations in their tails. They may even differ in the overall number of pulses. We show two such states at $R_{T}=1.23 R_{T}^{(0)}$ in Fig. 8 and summarize their properties in Table I. Other stable states have been found by starting from a stable state and either increasing or decreasing $R_{T}$. For example, at $R_{T}=1.18 R_{T}^{(0)}$ we found a single pulse using the numerically stable pattern found for $R_{T}=1.19 R_{7}^{(0)}$ as initial configuration, but three pulses when starting from the numerically stable pattern found for $R_{T}=1.15 R_{T}^{(o)}$ (not shown).

\section{Summary of transport properties}

The transport properties of the pulses are summarized in Tables I-III. In all cases the total thermal and solutal Nusselt numbers $N_{T}$ and $N_{S}$ are increasing functions of $R_{T}$. Within the states sharing the same Rayleigh number $\left(R_{T}\right.$ $\left.=1.23 R_{T}^{(0)}\right)$, the Nusselt numbers increase with the number of rolls in the state. The same holds for the reduced Nusselt numbers inside the pulses, $N_{T . p}$ and $N_{S, p}$, but not for the reduced Nusselt numbers in the background (see Table I), indicating a gradual increase in convection efficiency inside the pulses but not in the background region.

For $R_{T}=1.23 R_{T}^{(\rho)}$ the two states with 26 roll pairs and two pulses share almost identical transport properties, being differentiated only by the relative size of the pulses and their mutual separation. Despite these differences the total extent of the pulse and the background regions is roughly the same in both cases, with the pulse widths comparable in the first, and the two background regions comparable in the second.

When phase is conserved (as for $R_{T}=1.19 R_{T}^{(o)}, 1.23 R_{T}^{(o)}$, $1.27 R_{T}^{(n)}$ ), the pulses are very active dynamical structures that play a substantial role in convective transport. In this regime the proportion of the kinetic energy, of the enstrophy, and also of the vertical heat and solute transports attributed to the pulses exceeds the relative portion of the domain occupied by the pulses. Thus pulses enhance the overall transport properties of the system (cf. Table II). Moreover, when two pulses are present, the fraction of the domain they occupy increases both with $R_{T}$ and with the number of rolls in the pattern. In contrast, when phase is not conserved (as for $R_{T}=1.31 R_{T}^{(0)}$ and $1.35 R_{T}^{(0)}$ ) the proportional heat and solute transports by the pulses are comparable to the proportional extent they occupy in the horizontal, indicating that the presence of the pulses no longer increases transport efficiency.

In all cases studied the average velocity of the rolls $\left(\bar{v}_{r}\right)$ decreases with increasing Rayleigh number while the overall average roll length $\left(\bar{\lambda}_{r}\right)$ increases. The roll velocity within a pulse $\left(\bar{v}_{r, p}\right)$ also decreases with increasing Rayleigh number, while in states corresponding to the same Rayleigh number $\left(R_{T}=1.23 R_{T}^{(0)}\right)$ it also decreases with increasing number of rolls (cf. Table IV). The velocity of the pulses is much less than the phase velocity of the long-wavelength modulation seen at small Rayleigh numbers $\left(R_{T}=1.15 R_{T}^{(o)}\right)$, indicating that the pulses are fully nonlinear states.

Table III compares the lateral fluxes of mass, heat, and solute obtained by averaging the horizontal fluxes corresponding to 50 snapshots spanning five units of time. There is a small lateral mass flux $m$, which for the Rayleigh number values analyzed ranges between -0.01 and -0.06 , namely, opposite to the motion of the rolls. This lateral mass flux originates from an asymmetry and tilting of the rolls. The lateral transports of heat and solute are also directed toward the left in these cases. These lateral fluxes are small when compared to the vertical ones (as judged by the ratios $Q_{T} / N_{T}$ or $\left.Q_{S} / N_{S}\right)$, namely, between 60 and 120 times smaller for the heat flux, and between 25 and 60 times smaller for the solute flux (cf. Tables I and III). In all cases the lateral transports show large relative deviations and their 
TABLE I. The thermal and solutal Nusselt numbers computed for the whole domain $\left(N_{T}, N_{S}\right)$, within the pulses $\left(N_{T, p}, N_{S, p}\right)$, and in the background $\left(N_{T, b}, N_{S, b}\right)$. Numbers in parentheses indicate standard deviations.

\begin{tabular}{|c|c|c|c|c|c|c|c|c|}
\hline$R_{T} / R_{T}^{(0)}$ & Roll pairs & Pulses & $N_{T}$ & $N_{T, p}$ & $N_{T, b}$ & $N_{S}$ & $N_{S, p}$ & $N_{S, b}$ \\
\hline 1.15 & 26 & 0 & $\begin{array}{c}1.43 \\
\left(9.5 \times 10^{-5}\right)\end{array}$ & & & $\begin{array}{c}1.52 \\
\left(1.4 \times 10^{-4}\right)\end{array}$ & & \\
\hline 1.19 & 26 & 1 & $\begin{array}{c}1.56 \\
\left(4.7 \times 10^{-4}\right)\end{array}$ & $\begin{array}{c}1.99 \\
\left(1.7 \times 10^{-1}\right)\end{array}$ & $\begin{array}{c}1.46 \\
\left(3.5 \times 10^{-2}\right)\end{array}$ & $\begin{array}{c}1.71 \\
\left(1.0 \times 10^{-3}\right)\end{array}$ & $\begin{array}{c}2.32 \\
\left(5.5 \times 10^{-1}\right)\end{array}$ & $\begin{array}{c}1.56 \\
\left(1.1 \times 10^{-1}\right)\end{array}$ \\
\hline \multirow[t]{5}{*}{1.23} & $22^{\lrcorner}$ & 2 & $\begin{array}{c}1.57 \\
\left(2.1 \times 10^{-3}\right)\end{array}$ & $\begin{array}{c}2.06 \\
\left(1.9 \times 10^{-1}\right)\end{array}$ & $\begin{array}{c}1.39 \\
\left(6.5 \times 10^{-2}\right)\end{array}$ & $\begin{array}{c}1.73 \\
\left(4.2 \times 10^{-3}\right)\end{array}$ & $\begin{array}{c}2.42 \\
\left(5.9 \times 10^{-1}\right)\end{array}$ & $\begin{array}{c}1.48 \\
\left(2.0 \times 10^{-1}\right)\end{array}$ \\
\hline & $24^{\mathrm{a}}$ & 2 & $\begin{array}{c}1.62 \\
\left(2.7 \times 10^{-3}\right)\end{array}$ & $\begin{array}{c}2.13 \\
\left(6.5 \times 10^{-2}\right)\end{array}$ & $\begin{array}{c}1.39 \\
\left(2.8 \times 10^{-2}\right)\end{array}$ & $\begin{array}{c}1.81 \\
\left(5.6 \times 10^{-3}\right)\end{array}$ & $\begin{array}{c}2.54 \\
\left(2.1 \times 10^{-1}\right)\end{array}$ & $\begin{array}{c}1.48 \\
\left(8.7 \times 10^{-2}\right)\end{array}$ \\
\hline & 26 & 2 & $\begin{array}{c}1.67 \\
\left(3.4 \times 10^{-3}\right)\end{array}$ & $\begin{array}{c}2.18 \\
\left(1.2 \times 10^{-1}\right)\end{array}$ & $\begin{array}{c}1.38 \\
\left(6.6 \times 10^{-2}\right)\end{array}$ & $\begin{array}{c}1.89 \\
\left(7.5 \times 10^{-3}\right)\end{array}$ & $\begin{array}{c}2.63 \\
\left(3.7 \times 10^{-1}\right)\end{array}$ & $\begin{array}{c}1.47 \\
\left(2.1 \times 10^{-1}\right)\end{array}$ \\
\hline & $26^{a \cdot b}$ & 2 & $\begin{array}{c}1.67 \\
\left(2.6 \times 10^{-3}\right)\end{array}$ & $\begin{array}{c}2.17 \\
\left(8.9 \times 10^{-2}\right)\end{array}$ & $\begin{array}{c}1.38 \\
\left(4.6 \times 10^{-2}\right)\end{array}$ & $\begin{array}{c}1.89 \\
\left(6.1 \times 10^{-3}\right)\end{array}$ & $\begin{array}{c}2.62 \\
\left(2.8 \times 10^{-1}\right)\end{array}$ & $\begin{array}{c}1.47 \\
\left(1.4 \times 10^{-1}\right)\end{array}$ \\
\hline & $33^{\mathrm{a}}$ & 3 & $\begin{array}{c}1.88 \\
\left(2.3 \times 10^{-3}\right)\end{array}$ & $\begin{array}{c}2.24 \\
\left(1.2 \times 10^{-1}\right)\end{array}$ & $\begin{array}{c}1.44 \\
\left(1.6 \times 10^{-1}\right)\end{array}$ & $\begin{array}{c}2.20 \\
\left(5.2 \times 10^{-3}\right)\end{array}$ & $\begin{array}{c}2.75 \\
\left(3.7 \times 10^{-1}\right)\end{array}$ & $\begin{array}{c}1.53 \\
\left(5.2 \times 10^{-1}\right)\end{array}$ \\
\hline 1.27 & 32 & 2 & $\begin{array}{c}1.95 \\
\left(8.9 \times 10^{-3}\right)\end{array}$ & $\begin{array}{c}2.40 \\
\left(1.3 \times 10^{-1}\right)\end{array}$ & $\begin{array}{c}1.60 \\
\left(9.9 \times 10^{-2}\right)\end{array}$ & $\begin{array}{c}2.34 \\
\left(2.2 \times 10^{-2}\right)\end{array}$ & $\begin{array}{c}3.05 \\
\left(4.1 \times 10^{-1}\right)\end{array}$ & $\begin{array}{c}1.79 \\
\left(3.1 \times 10^{-1}\right)\end{array}$ \\
\hline 1.31 & $46^{\mathrm{c}}$ & 2 & $\begin{array}{c}2.45 \\
\left(1.8 \times 10^{-2}\right)\end{array}$ & $\begin{array}{c}2.55 \\
\left(1.0 \times 10^{-1}\right)\end{array}$ & $\begin{array}{c}2.26 \\
\left(2.0 \times 10^{-1}\right)\end{array}$ & $\begin{array}{c}3.16 \\
\left(2.7 \times 10^{-2}\right)\end{array}$ & $\begin{array}{c}3.41 \\
\left(3.3 \times 10^{-1}\right)\end{array}$ & $\begin{array}{c}2.73 \\
\left(6.3 \times 10^{-1}\right)\end{array}$ \\
\hline 1.35 & $45^{\circ}$ & 2 & $\begin{array}{c}2.50 \\
\left(2.4 \times 10^{-2}\right)\end{array}$ & $\begin{array}{c}2.58 \\
\left(1.3 \times 10^{-1}\right)\end{array}$ & $\begin{array}{c}2.40 \\
\left(1.8 \times 10^{-1}\right)\end{array}$ & $\begin{array}{c}3.27 \\
\left(3.6 \times 10^{-2}\right)\end{array}$ & $\begin{array}{c}3.53 \\
\left(4.2 \times 10^{-1}\right)\end{array}$ & $\begin{array}{c}2.94 \\
\left(5.6 \times 10^{-1}\right)\end{array}$ \\
\hline 1.55 & $25^{\mathrm{c}, d}$ & $?$ & $\begin{array}{c}2.68 \\
\left(4.7 \times 10^{-2}\right)\end{array}$ & & & $\begin{array}{c}3.49 \\
\left(7.3 \times 10^{-2}\right)\end{array}$ & & \\
\hline
\end{tabular}

aCorresponds to mutated states.

'This two-pulse, 26 roll pair state differs from the one shown in Fig. 2(b) in the relative size of each of the pulses and in the interpulse separation.

${ }^{\mathrm{c}}$ Phase is not conserved for these values of $R_{T}$. The number of roll pairs fluctuates or is not precisely defined; the value reported is the most frequent in the time interval analyzed.

${ }^{\mathrm{d}}$ Pulses are no longer seen for this value of $R_{T}$.

dependence on the Rayleigh number remains unclear.

\section{B. Anatomy of a pulse}

Thus far we have characterized our solutions either by means of snapshots at particular instants of time or by means of horizontal averages. We now describe the properties of comoving time averages of temperature, solute, stream function, and vorticity, as well as of the thermal and total buoyancy forces defined by

$$
f_{B}^{(T)}=R_{T} \Theta . \quad f_{B}^{(S)}=-R_{S} \Sigma, \quad f_{B} \equiv f_{B}^{(T)}+f_{B}^{(S)}=R_{T} \Theta-R_{S} \Sigma .
$$

Time averaging of spatiotemporal patterns has proved to be a successful tool in analyzing complicated pattern-forming systems. In some cases it can reveal regular structures underlying disordered ones, even well into spatiotemporally chaotic regimes [17]. Indeed Barten et al. [16,7] have used time averages of fields to deduce the role played by the concentration field in holding together two different coexisting domains.

Time averages can be performed in the laboratory frame or from the reference point of a moving observer. The former provide essentially the same information as the instantaneous horizontal averages of the preceding section, and will not be considered further. We denote the latter by \langle\rangle$_{v}$, with the subscript $v$ indicating the velocity at which the observer is moving. Carefully chosen time averages filter out small-scale features of the fields and reveal their long-range components. In particular, the time averages recorded by an observer comoving with the pulses allows one to infer what the pulses are actually "seeing" when they drift along the domain.

A series of 50 to 100 consecutive snapshots of the fields was taken at equal time intervals of 0.1 , spanning from five to ten units of time. These fields were suitably shifted and then averaged together. Thus, if $F(x, z, t)$ represents any of the fields, then

$$
\langle F(x, z)\rangle_{\nu} \equiv \frac{1}{\tau} \int_{0}^{\tau} F(x-v t, z, t) d t \approx \frac{1}{n} \sum_{j=1}^{n} F\left(x-v t_{j}, z, t_{j}\right) .
$$

The resulting structures were further smoothed with a tapered low-pass filter.

Figure 9 shows the time-averaged fields at $R_{T}=1.23 R_{T}^{(o)}$ (with 26 roll pairs in the pattern) as seen by a comoving observer traveling at the pulse velocity $v_{p}=5.42$. The average reveals clearly the pulse location. The temperature and solute time averages possess smooth gradient variations, from $T=S=1$ at the bottom, to $T=S=0$ on top. Inside the pulse region, the vertical gradients of temperature and solute are smaller at midlayer than in the surrounding fluid. Those 
TABLE II. The ratio $\Delta_{p} / \Gamma$ denotes the fraction of the domain occupied by the pulses. Shown also are the fractions of the kinetic energy $E_{p} / E$ and enstrophy $Z_{p} / Z$ within the pulse regions relative to those quantities for the whole domain, and the proportion of the vertical heat flux carried by the pulses compared to the total, namely, $N_{T, p} \Delta_{p} / N_{T} \Gamma$, and the analogous proportion of the vertical solute fux. Numbers in parentheses indicate standard deviations.

\begin{tabular}{cccccccc}
\hline \hline$R_{T} / R_{T}^{(o)}$ & Roll pairs & Pulses & $\Delta_{p} / \Gamma$ & $E_{p} / E$ & $Z_{p} / Z$ & $N_{T, p} \Delta_{p} / N_{T} \Gamma$ & $N_{S, p} \Delta_{p} / N_{S} \Gamma$ \\
\hline 1.19 & 26 & 1 & 0.19 & 0.29 & 0.32 & 0.24 & 0.26 \\
& & & $\left(6.3 \times 10^{-4}\right)$ & $\left(7.9 \times 10^{-4}\right)$ & $\left(3.2 \times 10^{-3}\right)$ & $\left(2.1 \times 10^{-2}\right)$ & $\left(6.1 \times 10^{-2}\right)$ \\
1.23 & $22^{\mathrm{a}}$ & 2 & 0.27 & 0.45 & 0.49 & 0.35 & 0.37 \\
& & & $\left(6.4 \times 10^{-3}\right)$ & $\left(1.1 \times 10^{-2}\right)$ & $\left(1.2 \times 10^{-2}\right)$ & $\left(3.3 \times 10^{-2}\right)$ & $\left(9.3 \times 10^{-2}\right)$ \\
& $24^{\mathrm{a}}$ & 2 & 0.31 & 0.51 & 0.55 & 0.41 & 0.44 \\
& & & $\left(8.1 \times 10^{-4}\right)$ & $\left(1.7 \times 10^{-3}\right)$ & $\left(2.6 \times 10^{-3}\right)$ & $\left(1.2 \times 10^{-2}\right)$ & $\left(3.6 \times 10^{-2}\right)$ \\
& 26 & 2 & 0.36 & 0.56 & 0.61 & 0.47 & 0.50 \\
& $266^{\mathrm{ab}}$ & 2 & $\left(8.3 \times 10^{-4}\right)$ & $\left(1.6 \times 10^{-3}\right)$ & $\left(4.4 \times 10^{-3}\right)$ & $\left(2.5 \times 10^{-2}\right)$ & $\left(6.9 \times 10^{-2}\right)$ \\
& & & $\left(1.4 \times 10^{-3}\right)$ & $\left(2.3 \times 10^{-3}\right)$ & $\left(4.2 \times 10^{-3}\right)$ & $\left(1.9 \times 10^{-2}\right)$ & $\left(5.4 \times 10^{-2}\right)$ \\
& $33^{\mathrm{a}}$ & 3 & 0.54 & 0.72 & 0.75 & 0.65 & 0.68 \\
& & & $\left(1.2 \times 10^{-3}\right)$ & $\left(4.2 \times 10^{-3}\right)$ & $\left(5.1 \times 10^{-3}\right)$ & $\left(3.3 \times 10^{-2}\right)$ & $\left(9.0 \times 10^{-2}\right)$ \\
1.27 & 32 & 2 & 0.44 & 0.60 & 0.63 & 0.54 & 0.57 \\
& \multirow{2}{*}{32} & & $\left(8.5 \times 10^{-3}\right)$ & $\left(1.3 \times 10^{-2}\right)$ & $\left(1.3 \times 10^{-2}\right)$ & $\left(2.9 \times 10^{-2}\right)$ & $\left(7.6 \times 10^{-2}\right)$ \\
1.31 & $46^{\mathrm{c}}$ & 2 & 0.63 & 0.62 & 0.65 & 0.66 & 0.68 \\
& & & $\left(2.2 \times 10^{-2}\right)$ & $\left(3.5 \times 10^{-2}\right)$ & $\left(3.2 \times 10^{-2}\right)$ & $\left(3.8 \times 10^{-2}\right)$ & $\left(7.1 \times 10^{-2}\right)$ \\
1.35 & $45^{\mathrm{c}}$ & 2 & 0.54 & 0.51 & 0.55 & 0.56 & 0.59 \\
& & & $\left(1.6 \times 10^{-2}\right)$ & $\left(2.1 \times 10^{-2}\right)$ & $\left(1.9 \times 10^{-2}\right)$ & $\left(3.4 \times 10^{-2}\right)$ & $\left(7.2 \times 10^{-2}\right)$ \\
\hline \hline
\end{tabular}

"Corresponds to mutated states.

${ }^{b}$ This two-pulse, 26 roll pair state differs from the one shown in Fig. 2(b) in the relative size of each of the pulses and in the interpulse separation.

'Phase is not conserved for these values of $R_{T}$. The number of roll pairs fluctuates or is not precisely defined; the value reported is the most frequent in the time interval analyzed.

same gradients are, however, very large close to the top and bottom boundaries, accounting for the larger reduced Nusselt numbers inside the pulses. For ease of comparison the total and thermal buoyancy force fields, as in Eq. (17), are plotted using the same set of isobuoyancy contours. Thermal and solutal (not shown) buoyancy forces possess rather featureless profiles, much like the temperature and solute averages, except for a couple of pronounced maxima and minima that reveal the location of the pulse. Both combine to give a complicated structure to the total buoyancy force in the pulse region.

The stream function panel in Fig. 9 shows an average long-range flow that circulates along the whole domain from right to left, with streamlines wiggling up and down through the bulk of the fluid. This is a familiar property of traveling wave streamlines in the comoving frame [18]. In the laboratory frame the magnitude of the lateral mean flow spanning the domain was too weak to reveal itself readily in the stream function contour plots [cf. Fig. 3(b)]. The striking feature of this averaged stream function is the presence of groups of vortices signaling the position of the pulses. There are two pairs of vortices for each pulse, one at the leading edge of the pulse and the other at the trailing edge, each pair with one vortex above and one below midlevel in the domain. The vortex pair at the trailing edge (on the left) is the strongest of the two, with the one at the top rotating counterclockwise and the lower one clockwise, both embraced by the flow lines of the long-range flow. The pair at the leading edge is weaker and covers a larger extent of the pulse area, with a clockwise vortex on top and a counterclockwise one at the bottom.

It is not surprising to find a cluster of vortices traveling with the pulses. We should recall that in all cases more efficient transport was achieved in the pulse region: the background might have stronger plumes, but the ones inside the pulse are closer together. The plumes provide a coherent means for transporting both heat and solute. These transports depend on the correlation between the vertical component of the velocity $w$ and the temperature and solutal fields $T$ and $S$. The pulses are therefore regions of enhanced correlation between these fields and also of enhanced coherence among the plumes. The resulting spatial nonuniformity implies the presence of large-scale horizontal gradients of $w$ and hence the presence of large-scale vorticity. These vortices cannot be seen in Fig. 2 because they are much weaker than the convection rolls.

\section{PHASE EQUATION APPROACH TO THE PULSE FORMATION PROBLEM}

The pulses described in Sec. III were found to be very stable against random noise perturbations added to the fields during the numerical simulation. When two pulses were present, their separation and propagation speeds were unaffected by this type of perturbation, as long as the noise intensity remained below certain threshold values. When the noise level is set above the threshold the pattern is destroyed and the process of roll formation resumes almost from 
TABLE III. Time-averaged lateral mass flux $m$, the lateral heat flux $Q_{T}$ which represents the vertical integral of $\overline{Q_{T}}(z)$, and similarly the lateral solute fux $Q_{S}$. Numbers in parentheses indicate standard deviations.

\begin{tabular}{|c|c|c|c|c|c|}
\hline$R_{T} / R_{T}^{(u)}$ & Roll pairs & Pulses & $m$ & $Q_{T}$ & $Q_{s}$ \\
\hline 1.15 & 26 & 0 & $\begin{array}{r}-2.77 \times 10^{-2} \\
\left(9.1 \times 10^{-5}\right)\end{array}$ & $\begin{array}{c}-1.47 \times 10^{-2} \\
\left(4.7 \times 10^{-5}\right)\end{array}$ & $\begin{array}{c}-4.70 \times 10^{-2} \\
\left(1.5 \times 10^{-4}\right)\end{array}$ \\
\hline 1.19 & 26 & 1 & $\begin{array}{c}-4.26 \times 10^{-2} \\
\left(1.2 \times 10^{-3}\right)\end{array}$ & $\begin{array}{c}-2.12 \times 10^{-2} \\
\left(5.3 \times 10^{-3}\right)\end{array}$ & $\begin{array}{c}-6.64 \times 10^{-2} \\
\left(2.6 \times 10^{-2}\right)\end{array}$ \\
\hline \multirow[t]{5}{*}{1.23} & $22^{\mathrm{a}}$ & 2 & $\begin{array}{c}-3.17 \times 10^{-2} \\
\left(3.2 \times 10^{-3}\right)\end{array}$ & $\begin{array}{c}-1.54 \times 10^{-2} \\
\left(1.2 \times 10^{-2}\right)\end{array}$ & $\begin{array}{c}-4.84 \times 10^{-2} \\
\left(6.3 \times 10^{-2}\right)\end{array}$ \\
\hline & $24^{\mathrm{a}}$ & 2 & $\begin{array}{c}-3.58 \times 10^{-2} \\
\left(3.3 \times 10^{-3}\right)\end{array}$ & $\begin{array}{r}-1.67 \times 10^{-2} \\
\left(1.6 \times 10^{-2}\right)\end{array}$ & $\begin{array}{c}-5.16 \times 10^{-2} \\
\left(7.3 \times 10^{-2}\right)\end{array}$ \\
\hline & 26 & 2 & $\begin{array}{c}-3.95 \times 10^{-2} \\
\left(8.4 \times 10^{-4}\right)\end{array}$ & $\begin{array}{c}-1.99 \times 10^{-2} \\
\left(5.3 \times 10^{-3}\right)\end{array}$ & $\begin{array}{c}-6.27 \times 10^{-2} \\
\left(2.1 \times 10^{-2}\right)\end{array}$ \\
\hline & $26^{a, b}$ & 2 & $\begin{array}{c}-3.95 \times 10^{-2} \\
\left(2.6 \times 10^{-3}\right)\end{array}$ & $\begin{array}{c}-1.99 \times 10^{-2} \\
\left(1.2 \times 10^{-2}\right)\end{array}$ & $\begin{array}{c}-6.29 \times 10^{-2} \\
\left(5.4 \times 10^{-2}\right)\end{array}$ \\
\hline & $33^{\mathrm{a}}$ & 3 & $\begin{array}{c}-5.64 \times 10^{-2} \\
\left(4.4 \times 10^{-3}\right)\end{array}$ & $\begin{array}{c}-2.72 \times 10^{-2} \\
\left(2.4 \times 10^{-2}\right)\end{array}$ & $\begin{array}{c}-8.45 \times 10^{-2} \\
\left(1.1 \times 10^{-1}\right)\end{array}$ \\
\hline 1.27 & 32 & 2 & $\begin{array}{c}-4.31 \times 10^{-2} \\
\left(4.3 \times 10^{-3}\right)\end{array}$ & $\begin{array}{c}-2.54 \times 10^{-2} \\
\left(2.1 \times 10^{-2}\right)\end{array}$ & $\begin{array}{c}-8.82 \times 10^{-2} \\
\left(7.7 \times 10^{-1}\right)\end{array}$ \\
\hline 1.31 & $46^{c}$ & 2 & $\begin{array}{c}-2.95 \times 10^{-2} \\
\left(2.4 \times 10^{-3}\right)\end{array}$ & $\begin{array}{c}-1.44 \times 10^{-2} \\
\left(1.8 \times 10^{-2}\right)\end{array}$ & $\begin{array}{c}-4.45 \times 10^{-2} \\
\left(6.2 \times 10^{-2}\right)\end{array}$ \\
\hline 1.35 & $45^{c}$ & 2 & $\begin{array}{c}-1.03 \times 10^{-2} \\
\left(2.4 \times 10^{-3}\right)\end{array}$ & $\begin{array}{c}-5.54 \times 10^{-3} \\
\left(1.1 \times 10^{-2}\right)\end{array}$ & $\begin{array}{c}-1.56 \times 10^{-2} \\
\left(4.0 \times 10^{-2}\right)\end{array}$ \\
\hline 1.55 & $25^{\mathrm{c}, \mathrm{d}}$ & $?$ & $\begin{array}{c}5.88 \times 10^{-2} \\
\left(4.5 \times 10^{-3}\right)\end{array}$ & $\begin{array}{c}2.94 \times 10^{-2} \\
\left(9.9 \times 10^{-3}\right)\end{array}$ & $\begin{array}{c}9.47 \times 10^{-2} \\
\left(2.5 \times 10^{-2}\right)\end{array}$ \\
\hline
\end{tabular}

Corresponds to mutated states.

this two-pulse, 26 roll pair state differs from the one shown in Fig. 2(b) in the relative size of each of the pulses and in the interpulse separation.

'Phase is not conserved for these values of $R_{T}$. The number of roll pairs fluctuates or is not precisely defined; the value reported is the most frequent in the time interval analyzed.

${ }^{\mathrm{d}}$ Pulses are no longer seen for this value of $R_{T}$.

scratch, without memory of its past state. In contrast, when an existing state is mutated in the manner described in Sec. III A 5 the system usually relaxes into a new stable state. The numerical evidence thus favors the coexistence of multiple stable states. In this section we seek to elucidate (a) the nature of an individual pulse (its shape, propagation speed, etc.), (b) the origin of the double-pulse states described in Sec. III, and (c) the apparent multiplicity of pulse states alluded to above.

\section{A. Kinematics}

In all three cases discussed in detail in Sec. III the phase of the pattern was found to be conserved, that is, the number of convecting rolls stayed constant through time, without merging or splitting of rolls. Our theoretical approach takes this observation as its starting point. We think of the traveling wave in the form $\Psi(k x-\omega t+\varphi(x, t), z)$, where $\varphi(x, t)$ is the phase of the wave. For a uniform wave train the phase is constant. However, a spatially dependent phase provides a convenient description of nonuniform wave trains with wave number $q(x, t)$ and frequency $\Omega(x, t)$ that depend on position,

$$
q=k+\frac{\partial \varphi}{\partial x}, \quad \Omega=\omega-\frac{\partial \varphi}{\partial t}
$$

The simulations indicate a uniformly moving pulse, moving with speed $v_{p}$. The modulation in wave number and frequency thus takes the form

$$
\varphi(x, t)=\varphi\left(x-v_{p} t\right)
$$

Thus

$$
q=k+\varphi^{\prime}, \quad \Omega=\omega+v_{p} \varphi^{\prime}
$$

where the prime denotes differentiation with respect to argument, i.e., $\varphi^{\prime}=\partial \varphi / \partial \xi, \xi=x-v_{p} t$. Since the simulations indicate that $\varphi(x, t)$ is defined for all $(x, t)$ we have the kinematic relation

$$
\frac{\partial q}{\partial t}+\frac{\partial \Omega}{\partial x}=0
$$

that expresses conservation of waves [19]. In the frame of the pulse this relation takes the form

$$
\frac{\partial q}{\partial t}-v_{p} \frac{\partial q}{\partial \xi}+\frac{\partial \Omega}{\partial \xi}=0
$$

If $|q-k| \ll 1,|\Omega-\omega| \ll 1$ while the pulse interfaces (leading or trailing) have $O(1)$ thickness the appearance of the pulse 
TABLE IV. Comparison of computed pulse velocity $v_{p}$ with values predicted from the jump condition (25). See Sec. III A for symbol definition. When phase is conserved, typical measurement uncertainties for wavelengths and phase velocities are \pm 0.01 , and for pulse velocities \pm 0.02 .

\begin{tabular}{ccccccccccc}
\hline \hline$R_{T} / R_{T}^{(o)}$ & Roll pairs & Pulses & $\bar{\lambda}_{r}$ & $\bar{\lambda}_{r, b}$ & $\bar{\lambda}_{r, p}$ & $\bar{v}_{r}$ & $\bar{v}_{r, b}$ & $\bar{v}_{r, p}$ & $v_{p}^{(m) a}$ & $v_{p}^{(p) b}$ \\
\hline 1.15 & 26 & 0 & 2.00 & & & 15.15 & & & $11.65^{\mathrm{c}}$ & \\
1.19 & 26 & 1 & 2.12 & 2.86 & 1.31 & 14.21 & 15.01 & 10.05 & 5.88 & 5.86 \\
1.23 & $22^{\mathrm{d}}$ & 2 & 2.20 & 3.72 & 1.42 & 14.03 & 15.27 & 9.33 & 5.66 & 5.66 \\
& $24^{\mathrm{d}}$ & 2 & 2.28 & 3.65 & 1.26 & 13.46 & 15.02 & 8.69 & 5.37 & 5.38 \\
& 26 & 2 & 2.38 & 3.68 & 1.21 & 13.11 & 15.05 & 8.57 & 5.42 & 5.41 \\
& $26^{\mathrm{d}, \mathrm{e}}$ & 2 & 2.38 & 3.71 & 1.19 & 13.11 & 15.07 & 8.52 & 5.44 & 5.43 \\
1.27 & $33^{\mathrm{d}}$ & 3 & 2.44 & 3.65 & 1.20 & 11.66 & 15.11 & 8.46 & 5.39 & 5.21 \\
1.31 & 32 & 2 & 2.45 & 3.43 & 1.11 & 10.66 & 12.83 & 7.24 & 4.49 & 4.57 \\
1.35 & $46^{\mathrm{f}}$ & 2 & 2.27 & 3.33 & 0.99 & 7.55 & 11.77 & 5.99 & 3.63 & 3.54 \\
\hline \hline
\end{tabular}

Measured pulse velocity values.

${ }^{\mathrm{b}}$ Predicted pulse velocity values.

'Corresponds to small-amplitude long-wavelength modulations.

${ }^{\mathrm{d}}$ Corresponds to mutated states.

'This two-pulse 26 roll pair state differs from the one shown in Fig. 2(b) in the relative size of each of the pulses and in the interpulse separation.

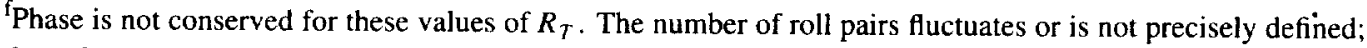
the value reported is the most frequent in the time interval analyzed.

is very abrupt and can then be viewed as a shock. Suppose that such a shock (again leading or trailing) forms at $\xi=\xi_{0}$ in the pulse frame. Integrating across the shock and noting that the shock is, by construction, stationary yields the "jump" condition

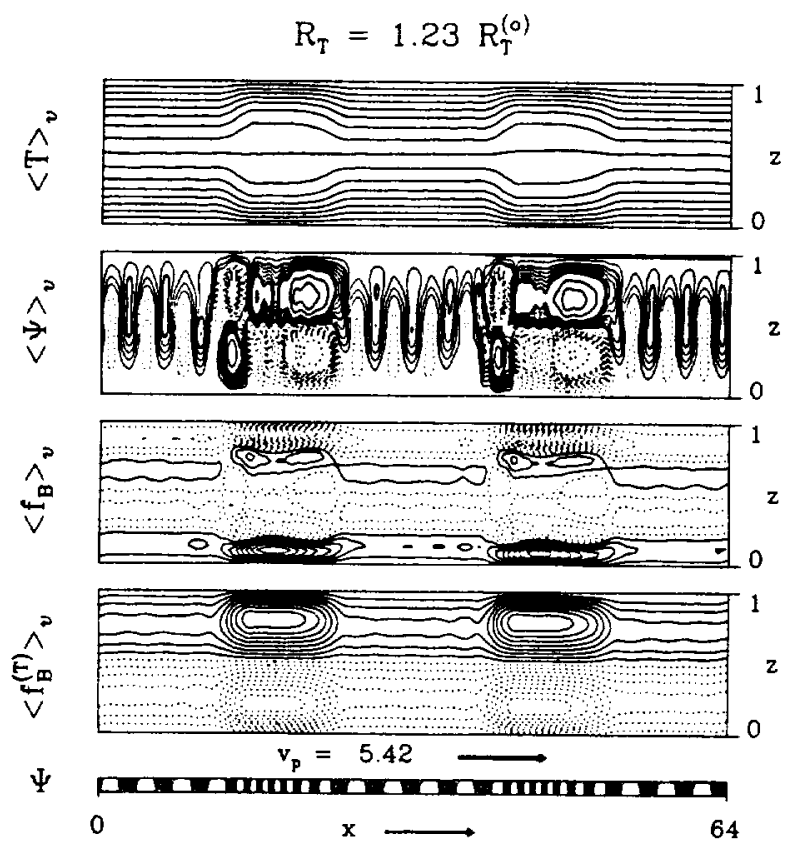

FIG. 9. Time-averaged fields in a frame traveling to the right with the pulse velocity for $R_{T}=1.23 R_{T}^{(o)}$. Shown are the averaged temperature $\langle T\rangle_{v_{p}}$, stream function $\langle\Psi\rangle_{v_{p}}$, total buoyancy force $\left\langle f_{B}\right\rangle_{v_{p}}$, and thermal contribution to the total buoyancy force $\left\langle f_{B}^{(T)}\right\rangle_{v_{p}}$, obtained from 100 snapshots spanning ten units of time. The beginning $\Psi$ field is included to specify the change in the vertical scale. The corresponding time average of the solutal concentration $S$ is similar to that of the temperature $T$ and has been omitted. The solutal contribution to the total buoyancy force $\left\langle f_{B}^{(S)}\right\rangle_{v_{p}}$ closely resembles $-\left\langle f_{B}^{(T)}\right\rangle_{v_{p}}$ and has also been omitted.

$$
v_{p} \Delta q=\Delta \Omega
$$

where $\Delta q \equiv q^{+}-q^{-}, \Delta \Omega \equiv \Omega^{+}-\Omega^{-}$represent the jumps in the wave number and frequency across the shock. We can rewrite this relation in the more convenient form

$$
v_{p}=\frac{\lambda^{+} v^{-}-\lambda^{-} v^{+}}{\lambda^{+}-\lambda^{-}},
$$

where $v \equiv \Omega / q$ is the local phase velocity and $\lambda \equiv 2 \pi / q$ the local wavelength. The predictions from this formula are compared with measured values of $v_{p}$ in Table IV. Since the pulses do not move completely rigidly [their widths measured by tracking regions where $\lambda_{r}(x)<\bar{\lambda}_{r}$ exhibit small oscillations due to the passage of rolls in and out of the pulse] the values of $v_{p}$ computed from Eq. (24) are not completely time independent. Consequently the values of $\bar{\lambda}_{r, b}$ and $\bar{v}_{r, b}$ listed in Table IV represent the average of their instantaneous values, measured frame by frame. Similarly, the measured pulse velocity $v_{p}$ also represents a time average. Nonetheless the average of the instantaneous values of $v_{p}$ computed from the jump condition (25) using the instantaneous values of $\bar{\lambda}_{p, b}$ and $\bar{v}_{p, b}$ shows remarkable agreement with the pulse velocity measured directly.

\section{B. Dynamics}

The phase $\varphi$ of the wave $\Psi(k x-\omega t+\varphi(x, t), z)$ satisfies an evolution equation called a phase equation. This equation follows from a gradient expansion $\left(\partial_{x} \varphi \ll 1, \partial_{t} \varphi \ll 1\right)$ and the symmetries of the underlying wave train. In the present case Galilean symmetry is absent (because of the no-slip boundary conditions imposed at $z=0,1$ ); consequently the wave train has only two symmetries, a continuous translation symmetry,

$$
\varphi \rightarrow \varphi+\text { const, }
$$


indicating the fact that the absolute value of the phase is arbitrary, and the discrete "mirror-glide" symmetry [2,1416]. If the wave train has period $2 \pi / k$ this symmetry acts by

$$
\Psi \rightarrow-\Psi, \quad \varphi \rightarrow \varphi+\frac{\pi}{k} .
$$

Consequently the mirror-glide symmetry has no effect on the structure of the phase equation. The most general evolution equation for the phase is therefore

$$
\begin{aligned}
\frac{\partial \varphi}{\partial t}+c_{g} \frac{\partial \varphi}{\partial x}= & -a \frac{\partial^{2} \varphi}{\partial x^{2}}-b \frac{\partial^{3} \varphi}{\partial x^{3}}-c \frac{\partial^{4} \varphi}{\partial x^{4}}-d\left(\frac{\partial \varphi}{\partial x}\right)^{2} \\
& -2 e \frac{\partial \varphi}{\partial x} \frac{\partial^{2} \varphi}{\partial x^{2}}-f\left(\frac{\partial \varphi}{\partial x}\right)^{3}-3 g\left(\frac{\partial \varphi}{\partial x}\right)^{2} \frac{\partial^{2} \varphi}{\partial x^{2}} \\
& -h\left(\frac{\partial^{2} \varphi}{\partial x^{2}}\right)^{2}-k \frac{\partial \varphi}{\partial x} \frac{\partial^{3} \varphi}{\partial x^{3}}-l\left(\frac{\partial \varphi}{\partial x}\right)^{4}+\cdots
\end{aligned}
$$

correct to fourth order in spatial derivatives, cf. [20]. Here $c_{g}$ is the group velocity of the perturbations at onset in the laboratory frame. This equation typically exhibits solutions in the form of spatiotemporal chaos, called phase turbulence [21]. Such spatiotemporal chaos occurs when $0<a \ll 1$, and is to be distinguished from the appearance of "phase slips" at which the phase description breaks down. These typically occur for larger values of $a$. As already mentioned, the presence of phase slips invalidates the phase description; this is not so when phase turbulence is present. In order to model the parameter regime $R_{T}=(1.19-1.27) R_{T}^{(0)}$ in which stable pulses are observed, and phase turbulence absent, we restrict the range of possible behavior by imposing the requirement that the right side of Eq. (28) be in conservation form, i.e., $d=f=l=0, h=k$. Thus

$$
\frac{\partial \varphi}{\partial t}+c_{g} \frac{\partial \varphi}{\partial x}=-\frac{\partial j}{\partial x}
$$

where

$$
j=a \varphi_{x}+b \varphi_{x x}+c \varphi_{x x x}+e \varphi_{x}^{2}+g \varphi_{x}^{3}+h \varphi_{x} \varphi_{x x}+\cdots .
$$

The motivation for studying Eq. (29) instead of Eq. (28) is threefold. First of all, it allows us to make contact with related work on confined states arising through phase instabilities of periodic patterns of steady rolls $[22,23]$. Second, it allows us to reduce the number of coefficients in the description. Finally, the phase equation in the pulse frame can be integrated once, yielding a third-order equation for the pulse shape. Given the fact that the observed pulses are characterized by a monotonic rise and an oscillatory trailing end such a description is the minimal one consistent with the observations. Moreover, as discussed further below, the observed pulse shapes are completely consistent with such a thirdorder description.

Several special cases of Eq. (29) have already been studied. For steady rolls the corresponding equation $\left(c_{g}=b=h\right.$ $=0$ ) is of $B-E$ type in the phase equation classification of Kuramoto [24]. The resulting equation has a Liapunov func- tional and consequently admits only time-independent states in the long time limit. This is also the case in the closely related Chapman-Proctor equation $\left(c_{g}=b=e=h=0\right)$ [25]. With appropriate boundary conditions all these equations have steady pulselike solutions, as discussed in detail by Deissler, Lee, and Brand [23]. However, because they have variational structure all stable pulses are necessarily subcriti$\mathrm{cal}$. This is because the equilibrium connected by the pulse must itself be stable. As a result the pulse formation process is hysteretic. In nonvariational systems this is not necessarily the case. Indeed pulses have been observed at supercritical parameter values in experiments on binary fluid convection [26]. Moreover Schöpf and Kramer [27] found stable pulses in the complex Ginzburg-Landau equation with a destabilizing cubic nonlinearity at supercritical values of the bifurcation parameter, while in systems with a nonzero group velocity pulses can also be stable in the supercritical regime, provided the basic state is only convectively, but not absolutely, unstable. This is the regime of interest in the present connection.

From Eq. (29) we see immediately that infinitesimal phase perturbations obey the dispersion relation

$$
s=a Q^{2}-c Q^{4}+\cdots+i Q\left(c_{g}-b Q^{2}+\cdots\right),
$$

where $\varphi(x, t) \sim \exp (s t-i Q x)$. Thus phase perturbations grow when $a Q^{2}-c Q^{4}+\cdots>0$. Since the anticipated instability is of long wavelength $(Q \ll 1)$ we suppose that $a$ and $c$ are both positive. The observed pulses are the outgrowth of the resulting convective instability $\left(0<a<2.12 \sqrt{b c_{g}}\right.$ if $b c_{g}>0,0$ $<a<\infty$ if $b c_{g}<0$, when $c=0$ ). We have seen that the instability evolves into a fully nonlinear pulse moving with speed $v_{p}$. Even though the phase equation (29) was obtained by a gradient expansion we shall find it to be useful as a model even for the fully nonlinear pulses for which $Q=O(1)$ seen in the simulations. A steadily moving pulse moving with speed $v_{p}$ to the right satisfies the equation

$$
\begin{aligned}
& \partial_{\xi}\left[\left(c_{g}-v_{p}\right) \varphi+a \varphi_{\xi}+b \varphi_{\xi \xi}+c \varphi_{\xi \xi \xi}+e \varphi_{\xi}^{2}+g \varphi_{\xi}^{3}+h \varphi_{\xi} \varphi_{\xi \xi}\right. \\
& +\cdots]=0 .
\end{aligned}
$$

Thus

$$
v \varphi+a \varphi_{\xi}+b \varphi_{\xi \xi}+c \varphi_{\xi \xi \xi}+e \varphi_{\xi}^{2}+g \varphi_{\xi}^{3}+h \varphi_{\xi} \varphi_{\xi \xi}+\cdots=0,
$$

where $v \equiv c_{g}-v_{p}$ and the constant of integration has been set to zero. In the following we think of Eq. (33) as a nonlinear eigenvalue problem for the speed $v$, and hence for the pulse speed $v_{p}$. As discussed further below the formulation of the boundary conditions for this problem depends on whether it is posed on the whole real line or on a finite (periodic) domain. If, in addition, the number of roll pairs in the perturbed and unperturbed wave trains is unchanged as a result of the formation of the pulse, there is no net phase jump $\Delta \varphi$ across the domain; this is not so if the number of roll pairs differs. The remaining boundary conditions for Eq. (33) are either that both $\varphi_{\xi}$ and $\varphi_{\xi \xi}$ vanish as $\xi \rightarrow \pm \infty$ (unbounded domain) or that both are periodic with period $\Gamma$ (bounded domain). 


\section{Pulses as homoclinic orbits}

We begin with the eigenvalue problem (33) on the real line, and use it to draw a number of general conclusions about the pulse shape and other properties. To this end we set $c=1$ and rewrite Eq. (33) as a dynamical system in $\xi$. We write $x$ for $\varphi$, so that Eq. (33) becomes

$$
\begin{gathered}
x^{\prime}=y, \\
y^{\prime}=z, \\
z^{\prime}=-v x-a y-b z-e y^{2}-g y^{3}-h y z,
\end{gathered}
$$

with the prime denoting $\partial / \partial \xi$. This dynamical system describes the spatial structure of the wave number modulation which is given by $y(\xi)$ in the present notation. It is this quantity that can be compared with our simulations. It is a dissipative system,

$$
\frac{\partial x^{\prime}}{\partial x}+\frac{\partial y^{\prime}}{\partial y}+\frac{\partial z^{\prime}}{\partial z}=-b-h y,
$$

with a single equilibrium at the origin corresponding to the unperturbed wave train.

Equation (34) is in the form of a particular codimensionthree bifurcation problem [28]. When $v=a=b=0$ the fixed point $(0,0,0)$ of Eq. (34) has three zero eigenvalues; the parameters $v, a, b$ can therefore be thought of as unfolding parameters that "unfold" this triple zero degeneracy. The pulses that are of interest correspond to homoclinic orbits of Eq. (34) that start from the origin at $\xi=-\infty$ and return to it at $\xi=+\infty$. The excursion of such an orbit (in $\xi$ space) away from the origin defines the shape of the pulse. Evidently, the question of whether such pulses exist is a global one. However, a necessary condition for the existence of a pulse is that $(0,0,0)$ is a generalized saddle in $\xi$, with at least one unstable eigenvector and at least one stable one.

The linearization of Eq. (33) around the origin yields the characteristic equation

$$
s^{3}+b s^{2}+a s+v=0
$$

where, for phase instability, $a>0$. Thus if $\operatorname{Re} s>0$ the solution grows with increasing $\xi$ but it decays with $\xi$ if $\operatorname{Re} s<0$. To construct a pulse both types of solution are necessary. Thus when $v>0$ we need either $b<0$ or $a b-v<0$ (or both) and when $v<0$ we need either $b>0$ or $a b-v>0$ (or both). The simplest situation arises when the phase modulations are only weakly dispersive so that $b \approx 0$. The sum of the eigenvalues $s_{1}, s_{2}, s_{3}$ is then zero, and hence either (i) $s_{1}=$ $-2 \mu, s_{2,3}=\mu \pm i \nu$, or (ii) $s_{1}=-\mu_{1}, s_{2}=-\mu_{2}, s_{3}=\mu_{1}$ $+\mu_{2}$. In the former case

$$
a=\nu^{2}-3 \mu^{2}>0, \quad 2 \mu\left(\mu^{2}+\nu^{2}\right)=v .
$$

This case applies when $v$ and hence $\mu$ are not too large, and is the case of greatest interest to us. Note, in particular, that $\operatorname{sgn} \mu=\operatorname{sgn} v$. Thus, when $v>0$, the origin has a onedimensional stable manifold and a two-dimensional unstable one; the opposite is the case when $v<0$. Hence when $v>0$ (i.e., $v_{p}<c_{g}$ ) the trajectory leaves $(0,0,0)$ at $\xi=-\infty$ as an exponentially growing oscillation; when it returns as $\xi \rightarrow \infty$ it does so as a decaying exponential without superposed oscillations. Thus the pulse shape is intrinsically asymmetric: when $v_{p}<c_{g}$ the leading edge of the pulse is monotonic while its trailing edge is an exponentially decreasing oscillation. The opposite is the case when $v_{p}>c_{g}$, in which case the pulse leads with an oscillatory precursor. In case (ii) which applies for larger $|v|$, e.g., for $v_{p} \ll c_{g}\left(v_{p} \gg c_{g}\right)$, the oscillatory tail (precursor) is absent, with the interface replaced by a monotonic one. The pulse remains generically asymmetric, however. These conclusions continue to hold when $0<|b| \ll 1$ and

$$
\begin{gathered}
s_{1}=-2 \mu-\frac{4 \mu^{2} b}{9 \mu^{2}+\nu^{2}}+O\left(b^{2}\right), \\
s_{2,3}=\mu-\frac{5 \mu^{2}+\nu^{2} b}{9 \mu^{2}+\nu^{2}} \frac{2}{2} \\
\pm i \nu\left(1+\frac{3 \mu^{2}-\nu^{2}}{9 \mu^{2}+\nu^{2}} \frac{\mu b}{2 \nu^{2}}\right)+O\left(b^{2}\right) .
\end{gathered}
$$

The numerical simulations provide valuable information about the pulse shape, and in particular about the wave number and frequency modulation due to the presence of a pulse. In Fig. 10 we show the wavelengths and phase velocities of the convection rolls at $R_{T}=1.19 R_{T}^{(o)}, R_{T}=1.23 R_{T}^{(o)}$, and $R_{T}=1.27 R_{T}^{(o)}$ at one instant in time. Observe that the wavelength and phase velocity curves have similar appearance. Both inside the pulses and outside the phase velocity and wavelength distributions are piecewise constant, with fairly rapid transitions between two sets of values, and both curves resemble one another. For pulses on the real line the phase velocity is defined as $v \equiv \Omega(x, t) / q(x, t)$ while the wavelength $\lambda \equiv 2 \pi / q(x, t)$. For small $\varphi^{\prime}$ it then follows that

$$
v-v_{p}=\left(v_{0}-v_{p}\right) \frac{\lambda}{\lambda_{0}},
$$

where $v_{0}$ and $\lambda_{0}$ are the phase velocity and wavelength of the background uniform wave train, i.e., $v_{0}=\omega / k, \lambda_{0}$ $=2 \pi / k$. Thus the phase velocity and wavelength are related linearly when phase gradients are small. Since $v_{0}-v_{p}>0$ (see Table IV) Eq. (39) indicates that the two curves should match without reflection. Figure 10 supports these conclusions; departures from the linear relation visible with increasing $R_{T}$ are not surprising since the phase gradients increase with increasing $R_{T}$.

The comparison of Fig. 10 with the long-wavelength theory thus clearly favors our case (i) with $v>0$. The single pulse present at $R_{T}=1.19 R_{T}^{(o)}$ leads with a monotonic front while its trailing edge decays exponentially at a different rate, with small superposed oscillations clearly visible. In the case of the two pulses found at $R_{T}=1.23 R_{T}^{(o)}$ both pulses have the same structure, with the oscillatory tail of the leading pulse decaying towards negative $\xi$ before turning into the leading front of the trailing pulse. At $R_{T}=1.27 R_{T}^{(0)}$ these oscillations are even more prominent, suggesting that $\mu$ is decreasing with $R_{T}$ and hence that the pulse velocity becomes closer to the linear group velocity. 

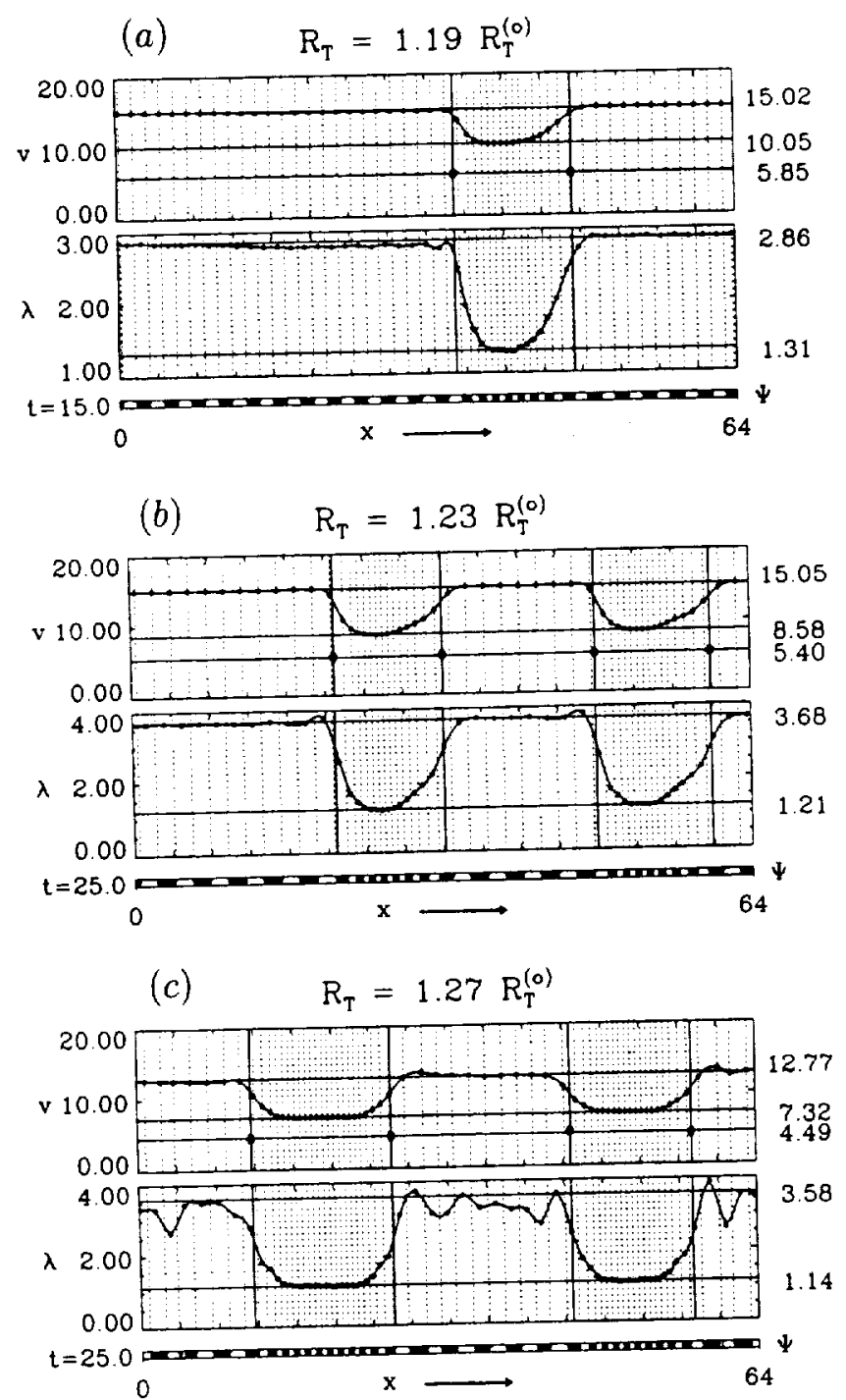

FIG. 10. Profiles of roll velocities and wavelengths as a function of position in the domain at one instant in time. The profiles travel almost rigidly to the right with speed $v_{p}$. Vertical lines indicate roll boundaries (dotted), and pulse boundaries (solid). The dots corre spond to computed values of wavelengths and phase velocities. Values at the right of the phase velocity panels are, from top to bottom, the phase velocities in the background and inside the pulse, and pulse velocity $v_{p}$. Values at the right of the wavelength panels are (top) the roll wavelengths outside the pulse and (bottom) inside the pulse. (a) $R_{T}=1.19 R_{T}^{(0)}$. (b) $R_{T}=1.23 R_{T}^{(0)}$. (c) $R_{T}=1.27 R_{T}^{(0)}$.

However, despite this superficial agreement with the longwave theory, the relation between the theoretical description of a pulse on the real line and a train of periodic pulses such as obtained in our numerical simulations is not straightforward. This is because in a finite domain with periodic boundary conditions the pulse is a fully nonlinear state everywhere: even in the wings of the pulse the wave number modulation about the putative background state does not vanish. Thus the use of periodic boundary conditions has important implications for the correct definition of the background state. To see this note that on the real line the definition of $\varphi(x, t)$ as the perturbation to the phase of a spatially uniform wave train and its localized nature imply that $\int_{-\infty}^{\infty} \varphi_{\xi} d \xi=\varphi(\infty)$ $-\varphi(-x)=0$. Thus the area under the wave number pertur- bation must be zero. On a finite but periodic domain the observed conservation of phase during the evolution of the phase instability likewise implies that $\int_{0}^{\Gamma} \varphi_{\xi} d \xi=\varphi(\Gamma)$ $-\varphi(0)=0$. As can be seen from Fig. 10 the condition $\int_{0}^{1} \varphi \xi d \xi=0$ defines a background (the underlying uniform wave train) that differs from the background wave number $k_{b}$ defined by the condition that $\left|\varphi_{\xi}-k_{b}\right| \ll 1$ in the largest possible fraction of the domain $0 \leqslant \xi \leqslant \Gamma$. Relative to this background $\int_{0}^{\Gamma}\left(\varphi_{\xi}-k_{b}\right) d \xi=A>0$, and hence $k_{b}=-A / \Gamma$ $<0$. The wave number shift $k_{b}$ is finite because the phase perturbation is distributed over a finite domain and not the whole line; $k_{b}$ vanishes as $\Gamma \rightarrow \infty$. Relative to the shifted background a pulse represents a net phase change, measured by $A$. In the following we therefore relate the observed pulses to solutions of Eq. (33) on $0 \leqslant \xi \leqslant \Gamma$ with the boundary conditions $\varphi(0)=0, \varphi(\Gamma)=A, \varphi_{\xi}$ and $\varphi_{\xi \xi}$ both periodic, with $A$ determined from Fig. 10. Similar issues were encountered already by Deissler, Lee, and Erand [23] in their study of confined states described by phase equations with variational structure.

\section{Determination of the dispersion relation}

In this section we use the shape of the pulse wings in Fig. 10 to determine the coefficients $a / c, b / c$, and $v / c$ in the dispersion relation (31) on the assumption that the observed pulses approximate the homoclinic pulses described in the preceding section. In view of the finite area $A$ and the $O(1)$ wavelength modulation in the observed pulses it is necessary that we first redefine the background wave number as discussed above. To determine the dispersion relation governing small wave number perturbations about this background we fit each interpulse region to a curve of the form

$$
f(x)=a_{0}+a_{1} x+a_{2} e^{\lambda_{1} x}+e^{\lambda_{2} x}\left[a_{3} \cos \left(\sigma_{2} x\right)+b_{3} \sin \left(\sigma_{2} x\right)\right],
$$

connecting the leading edge of one pulse to the trailing end of the preceding one. In cases where multiple pulses were present we focused on the largest interpulse region. We chose this approach instead of studying each pulse wing individually because the characteristics of adjacent pulses typically overlap. Even in the one-pulse case, where one would expect that the extent of the background region would make the leading and trailing pulse ends disconnected, the wiggling of the trailing end propagates all the way up to the leading end. In all cases the fitted value of $a_{0}$ was very close to the measured wavelength of the rolls in the background. The parameter $a_{1}$ turned out to be zero in all the fits except in the one-pulse case $\left(R_{T}=1.19 R_{T}^{(0)}\right)$ in which a slight slope of the background is present, representing a wave number gradient between the pulse and its periodic image. The fitted values of $\lambda_{1}, \lambda_{2}$, and $\sigma_{2}$ provide estimates of the quantities $s_{1}, \operatorname{Re} s_{2}$, and $\operatorname{Im} s_{2}$, respectively, and hence of the roots $s_{1}$, $s_{2}, \bar{s}_{2}$ of the characteristic equation (36). Table $\mathrm{V}$ lists these estimates. Such estimates improve with the interpulse separation. In a successful fit $\lambda_{1}<0, \lambda_{2}>0$. From these eigenvalues we can obtain values of $a, b$, and $v$. To check on the consistency of these results we use the observed pulse speed $v_{p}$ to deduce from $v$ the group velocity $c_{z}$. Table VI lists the 
TABLE V. Exponential fits to the largest interpulse intervals, computed by fitting the function (40) to the measured data by least squares techniques. Numbers in parentheses indicate standard deviations.

\begin{tabular}{cccc}
\hline \hline$R_{T} / R_{T}^{(o)}$ & $s_{1}$ & $\operatorname{Re} s_{2}$ & $\operatorname{Im} s_{2}$ \\
\hline 1.19 & -0.82 & 1.36 & 0.90 \\
& $(0.08)$ & $(0.43)$ & $(0.61)$ \\
1.23 & -0.82 & 1.52 & 1.05 \\
& $(0.45)$ & $(0.38)$ & $(0.46)$ \\
$1.23^{\mathrm{a}}$ & -0.72 & 1.75 & 1.12 \\
& $(0.11)$ & $(0.51)$ & $(0.41)$ \\
$1.23^{\mathrm{b}}$ & -0.92 & 1.63 & 0.74 \\
& $(0.14)$ & $(0.29)$ & $(0.48)$ \\
$1.23^{\mathrm{c}}$ & -1.02 & 1.51 & 1.18 \\
& $(0.19)$ & $(0.65)$ & $(1.28)$ \\
1.27 & -0.75 & 1.17 & 0.76 \\
& $(0.09)$ & $(0.69)$ & $(0.77)$ \\
\hline \hline
\end{tabular}

${ }^{a}$ Two-pulse mutated state with 26 roll pairs.

b Two-pulse mutated state with 24 roll pairs.

${ }^{c}$ Two-pulse mutated state with 22 roll pairs.

resulting values of $a, b, v$, and $c_{g}$. There is no indication that a higher-order characteristic equation would lead to an improvement in these fits.

In all cases the fitted values of $v$ are positive, implying that the pulse (or pulse pair) travels more slowly than the group velocity $c_{g}$ for long-wavelength perturbations. Moreover, in these cases the fitted values of $a$ are positive, implying that the basic wave train is unstable with respect to longwavelength phase instabilities, as hypothesized. A significant source of error arises from fits that put too much weight on points that are contaminated by nonlinear effects; this problem increases with decreasing interpulse distance and hence becomes particularly acute for multipulse states. In such states the double-pulse solution of the dynamical system (34) with the appropriate period may not approach the origin closely enough to sample the linear dispersion relation that describes its behavior in its vicinity. Consequently we expect that the values of the dispersion relation coefficients deduced from the corresponding fits will be less accurate than those obtained in the single-pulse case. This may account for some of the scatter in the coefficient values, particularly in $a$.

TABLE VI. The values of the dispersion relation coefficients $a$, $b$, and $v$ in Eq. (36) obtained from the fitted values of the eigenvalues $s$.

\begin{tabular}{lcccc}
\hline \hline$R_{T} / R_{T}^{(o)}$ & $a$ & $b$ & $v$ & $c_{g}$ \\
\hline 1.19 & 0.42 & -1.90 & 2.21 & 8.09 \\
1.23 & 0.91 & -2.21 & 2.78 & 8.20 \\
$1.23^{\mathrm{a}}$ & 1.79 & -2.78 & 3.10 & 8.54 \\
$1.23^{\mathrm{b}}$ & 0.22 & -2.35 & 2.94 & 8.31 \\
$1.23^{\mathrm{c}}$ & 0.61 & -2.00 & 3.73 & 9.39 \\
1.27 & 0.21 & -1.60 & 1.46 & 5.95 \\
\hline \hline
\end{tabular}

Two-pulse mutated state with 26 roll pairs

${ }^{\mathrm{b}}$ Two-pulse mutated state with 24 roll pairs.

${ }^{c}$ Two-pulse mutated state with 22 roll pairs.
These results parallel a recent measurement of the phase diffusion coefficient for traveling wall modes in rotating convection [29]. See also [20].

The last column in Table VI lists the values of the group velocity deduced from the values of $v$ in the previous column with the help of the results listed in Table IV. The rough consistency of these values is reassuring, and supports our interpretation of the pulse formation process as the consequence of a long-wavelength phase instability and its description using the third-order phase equation (29). Note that such consistency could not hold if we fitted each pulse separately. Such individual fits would yield a value of $v$ but this value would reflect the speed of the pulse only in the absence of all others. In fact the pulse pairs travel together with a common (constant) speed, determined by the pulse-pulse interaction, and it is this speed that is determined by fitting the largest interpulse interval. This proviso applies to the single pulse as well: because of the use of periodic boundary conditions a single-pulse state is in fact a periodic train of equally spaced pulses. Such a pulse train has a different speed than an isolated pulse of identical shape.

\section{E. Pulse multiplicity and the Shil'nikov mechanism}

The inference that Eq. (29) possesses a homoclinic orbit of saddle-focus type for appropriate values of the coefficients of the nonlinear terms has a number of additional consequences. A general theory of the dynamics (in $\xi$ ) when such orbits are present is due to Shil'nikov [30]; its elaboration by Glendinning and Sparrow [31] is particularly useful. The theory defines a quantity $\delta$ (the eigenvalue ratio) as

$$
\delta=-\frac{\operatorname{Re} s_{2}}{s_{1}}
$$

and shows that a homoclinic orbit to a fixed point with $\delta$ $>1$ is generically simple and isolated. On the other hand, if $\delta<1$, the orbit coexists with an uncountable number of nonperiodic orbits as well as a countable number of periodic orbits of arbitrarily large periods. Although all these orbits correspond to different spatial states they do not resemble the observed pulselike states. However, Glendinning and Sparrow show that for parameters near those required for the formation of the primary (single-pulse) homoclinic orbit there are other parameter values (i.e., values of $v$ ) for which double-pulse homoclinic orbits are present. These homoclinic orbits (called secondary by Glendinning and Sparrow) start out as if to form a single pulse but miss the origin the first time around, and so spiral away from it again, forming a second pulse. Only after a second large-amplitude excursion from the origin do they connect to the origin. Such solutions (see Fig. 11) are exactly of the form of our twopulse states. Note that our approach predicts that a double pulse should move at constant speed given by the corresponding eigenvalue $v$, maintaining constant separation between the two pulses. As suggested in Fig. 11 there is in fact a countably infinite number of such double-pulse (also triplepulse etc.) states which accumulate (exponentially) on the parameter value, $v^{*}$ say, at which the primary one-pulse homoclinic orbit is present. These orbits differ in the number of turns each makes around the origin: the outer ones $(v$ 


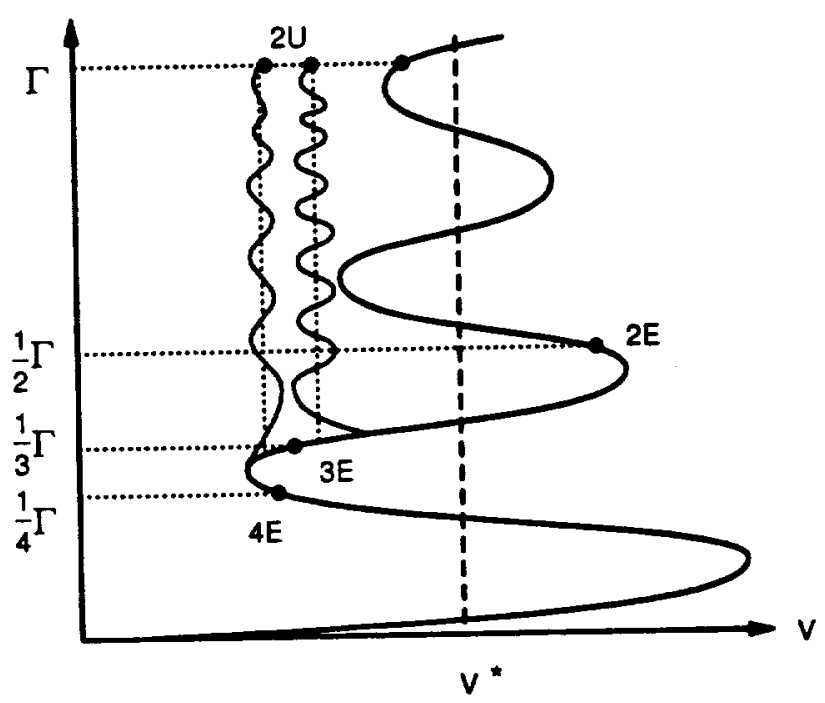

FIG. 11. The modulation wavelength $\Lambda$ vs $v$ showing the creation of single- and multiple-pulse states near the primary homoclinic connection at $v^{*}$ (corresponding to a single pulse on the real line), satisfying the Shil'nikov condition $\delta<1$.

$-v^{*}$ large) perform few turns and hence correspond to double pulses with few oscillations in $q$ separating them, while the inner ones perform more and more such oscillations as their eigenvalue $v$ approaches $v^{*}$ (see Fig. 11). The double-pulse homoclinic orbits accumulate on $v^{*}$ at a geometric rate given by, cf. [32],

$\frac{v_{n+1}}{v_{n}}=\exp \left(-\frac{2 \pi \mu}{\nu}+O(b)\right)=\exp \left(-\frac{2 \pi \mu}{\sqrt{a+3 \mu^{2}}}+O(b)\right)$

where $v^{*}=2 \mu\left(a+4 \mu^{2}\right)$. In contrast the saddle-node bifurcations (see Fig. 11) accumulate at the rate

$$
\begin{aligned}
\frac{v_{n+1}}{v_{n}} & =-\exp \left(-\frac{\pi \mu}{\nu}+O(b)\right) \\
& =-\exp \left(-\frac{\pi \mu}{\sqrt{a+3 \mu^{2}}}+O(b)\right),
\end{aligned}
$$

It should be noted that no similar behavior occurs in case (ii) in which only the primary one-pulse orbit is present at $v^{*}$, cf. [33]; as $v \rightarrow v^{*}$ the period of a periodic array of pulses increases monotonically with no "horseshoe" formation, much as in case (i) with $\delta>1$.

Thus far we have discussed the consequences of having a homoclinic orbit to a saddle focus satisfying the Shil'nikov condition $0<\delta<1$. This theory applies on the real line $-\infty$ $<\xi<+\infty$. Our numerical simulations use a finite albeit large domain with periodic boundary conditions. Consequently, true homoclinic pulses cannot form, and our pulse states must be interpreted as periodic states with a long but finite period. In particular, the plethora of nonperiodic trains of pulses that coexist with the primary pulse at $v^{*}$ cannot be realized in our periodic geometry. Among the periodic states present at $v^{*}$ only those with the correct period can be realized. We indicate this process by the dashed horizontal lines drawn in Fig. 11 which correspond to the period $\Gamma$ and its integer fractions $\Gamma / N, N=1,2, \ldots$. As the wavelength curve $\Lambda(v)$ approaches the primary homoclinicity at $v^{*}$ it is cut by the dashed lines. The intersection with the line $\Gamma / N$ indicates the presence of a single-pulse state with period $\Gamma / N$ at the corresponding $v$. This state can be replicated forming an $N$-pulse state that fits within the full period $\Gamma$. Such multipulse states are characterized by equal separation between the constituent pulses. Figure 11 also shows one of infinitely many subsidiary homoclinicities corresponding to the formation of double pulses. The speed of such pulses corresponds to the intersection of the corresponding $\Lambda(v)$ loci with the line $\Gamma$. These double-pulse states are fundamentally different because while they still move rigidly with the speed given by this intersection their separation will be uneven. Moreover, as indicated in Fig. 11, the different double states formed this way will have different numbers of oscillations in their tails and will propagate with slightly different speeds than pairs of identical pulses. Figures 2(b) and 8(b) illustrate two distinct solutions, both with 26 roll pairs and two pulses with uneven pulse separation. In a similar way one can construct a variety of three-pulse states [see Fig. 8(a)]. However, recent theoretical work [34] indicates that such constructions are not of finite codimension; in particular, on the real line three-pulse states can be produced by arbitrarily small changes in the eigenvalue ratio $\delta$. Consequently any attempt to describe the observed three-pulse states by a truncated dynamical system of the form (34), i.e., a truncated phase equation, is doomed to fail.

In the theory the case $\delta=\frac{1}{2}$ is a degenerate one because the linearization about the origin is divergence-free. This is the case for the Kuramoto-Sivashinsky equation [35]. In the present case when dispersion $(b \neq 0)$ is included we find that

$$
\delta=\frac{1}{2}\left(1-\frac{b}{2 \mu}\right)+O\left(b^{2}\right)
$$

Thus the Shil'nikov mechanism is generic in the present problem only in the presence of dispersion.

\section{F. Pulse solutions of the phase equation}

The conclusions we have drawn so far are predicated on the presence of a homoclinic orbit of saddle-focus type in Eq. (29) when the coefficients of the linear terms take the values deduced from the observed pulse shapes. But whether such an orbit is present is clearly a function of the nonlinear terms. It is a simple matter to show that for some choices of the coefficients the requisite homoclinic orbit cannot exist. For example, when $v>0, b<0$ (and $A=0$ ) this is the case for all $h \leqslant 0$. Evidently a more detailed theory would start with known (calculated) values of the coefficients $a, \ldots, h$, and ask for what value (or values) of $v$ do such orbits exist. Such a theory would not only predict the shape of the pulse but also its speed, rather than simply looking for consistency in the general picture. Since we are not in a position to complete this program we simply confirm that the phase equation (29) is capable of describing pulse states of the type described in Sec. III. To this end we have solved the nonlinear eigenvalue problem (33) on a finite domain of length $\Gamma$ $=64$ with a discretized one-dimensional mesh using an iterative Newton-Raphson-Kantorovich scheme [36] of high accuracy in both the $L_{2}$ norm of $\varphi_{\xi}$ and the corresponding 

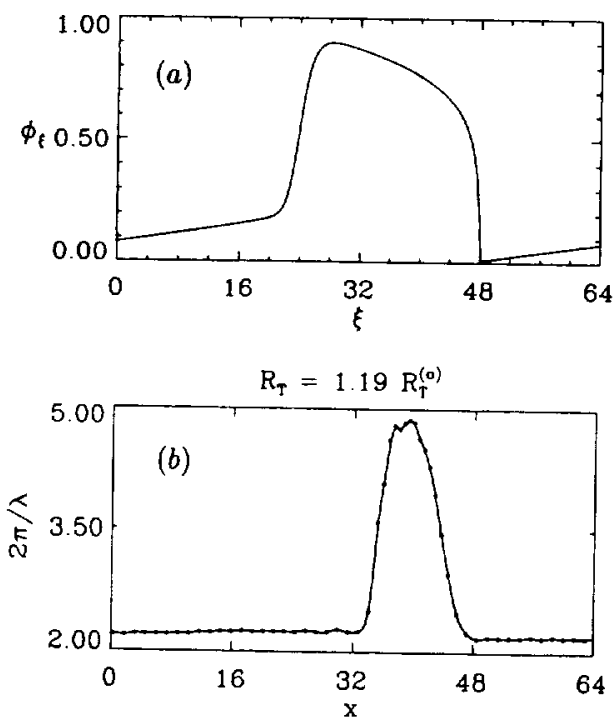

FIG. 12. (a) Solution $\varphi_{\xi}(\xi)$ of the phase equation (33) with $a$ $=0.4, b=-1.9, c=1.0, e=-800, g=820, h=700$, and an imposed phase jump $A=3.63 \times 2 \pi$. The eigenvalue $v=6.20$ implies a pulse speed $v_{p} \approx 1.88$. (b) The instantaneous local wave number $q(x) \equiv 2 \pi / \lambda(x)$ computed from Fig. 10(a) for comparison with (a).

eigenvalue $v$. For the linear coefficients we used the values deduced from the pulse shape in Sec. IV D, $a=0.4, b=$ $-1.9, c=1.0$; in an effort to model the single pulse observed at $R_{T}=1.19 R_{T}^{(o)}$ we used $\varphi(0)=0, \varphi(\Gamma)=A$, as the boundary conditions, with $\varphi_{\xi}, \varphi_{\xi \xi}$ periodic and $A$ determined from Fig. 10(a). The result shown in Fig. 12(a) is for $e<0$ and $g>0$ for which homoclinic orbits to a nonzero background wave number are possible. The figure shows a single pulse in terms of the wave number modulation $\varphi_{\xi}(\xi)$, riding on a sloping background corresponding to a nonuniform background wave number. In Fig. 12(b) we show, for comparison, Fig. 10(a) redrawn in terms of the local wave number. For the parameter values chosen, the solution of the phase equation yields a broader pulse than realized in the simulation. As discussed in Sec. IV D the wave number gradient was found to be an essential part of the fitting procedure [Eq. (40)]. The asymmetry of the pulse in Fig. 12(a) is similar to that of the observed pulse, while the corresponding eigenvalue $v=6.20$ yields a pulse speed $v_{p}$ that is about one-third of the observed one.

The phase equation (29) exhibits a rich variety of pulselike solutions which will be described elsewhere. The formulation of the pulse formation problem on a finite domain facilitates the location of such solutions, but requires the specification of the phase jump $A$ across the domain. The finite size of the domain tends to suppress phase turbulence, and extends the parameter regime in which the phase equation exhibits pulselike solutions.

\section{G. Stability considerations}

Thus far we have not addressed the stability properties of the various states identified above as solutions of the phase equation (29). We plan to give a detailed discussion of these and of the multiplicity of pulselike solutions to Eq. (29) elsewhere. However, the following remarks can be made.
We have taken the point of view that the states found in the numerical simulations of Eqs. (1)-(5) are stable, although this is in fact something that is extremely hard to demonstrate, both mathematically and numerically (because of the very long horizontal diffusion times across the domain). Any stability discussion divides naturally into two parts, the stability of a single-pulse state, and the stability of a periodic train of pulses. Assuming that only one pulse is present per period one first examines the stability of this state with respect to perturbations with the same wavelength; subsequently one can examine the stability with respect to perturbations with periods that are increasing multiples of the basic period. Unfortunately the stability properties of homoclinic pulses on the whole real line are not easily established, even in dissipative systems [37]. In fact we expect that most such states will prove to be unstable, possibly including the one shown in Fig. 12(a). Usually stable pulses on the real line require that the equilibrium state that is connected by the pulse is stable; otherwise the pulse inherits the instability of the equilibrium. Thus stable pulses are usually subcritical. In our case this argument suggests that stable pulses should only be observed when the phase diffusion coefficient $-a$ is positive. While this is so for the Eckhaus instability, in dispersive systems, such as the one considered here, stable pulses can be present even when the basic equilibrium is unstable, provided that the instability is only convective. In fact the theory described above applies equally well for $a$ negative as for $a$ positive. The only difference is that when $a<0$ the quantity $\mu$ in Eq. (37) must be sufficiently large. It follows that $v$ must also be sufficiently large and hence that the pulse speed $v_{p}$ will differ substantially from the group velocity $c_{g}$. In fact this is the case for the pulses we have found (see Table IV). However, we have been unable to find any signature of the required hysteresis in the pulse formation process and consequently favor the choice $a>0$. Assuming that a single pulse per wavelength is stable the stability of a whole train of such pulses may then be described as in Refs. [38,39]. One finds that instability that leads to pulse bunching (i.e., a nonuniform train of pulses) depends on the dispersion relation giving the pulse speed $v_{p}$ as a function of the basic period or wavelength. Unfortunately since all our calculations were done with the same aspect ratio $\Gamma=64$ we have no information about this dispersion relation and hence about the stability properties of whole trains of pulses.

Finally, it is of interest to note that the phase equation examined by Janiaud et al. [20] also exhibits the formation of pulses, although in a rather limited parameter regime located astride the Benjamin-Feir instability boundary and very close to it. In the equation studied here the pulse formation process appears to be more robust, while its special form facilitates analytical approach.

\section{DISCUSSION AND CONCLUSIONS}

In this paper we have initiated a study of the mechanisms that lead to the formation of confined states in twodimensional large-aspect-ratio thermosolutal convection. $\mathrm{Nu}$ merical simulation of the Boussinesq equations describing the system in a two-dimensional periodic domain with realistic boundary conditions revealed the existence of a plethora 
of confined states, both single pulse and multipulse, all of which were found to travel rigidly on top of a uniform train of fully nonlinear traveling waves. All of our pulses traveled in the same direction as the underlying wave train but did so more slowly, sometimes substantially so, and appeared to be created spontaneously as a result of a phase instability. In particular, no finite-amplitude perturbations were required to form these pulses, and no evidence of hysteresis in their formation was uncovered. The pulses lead to the appearance of spatially nonuniform wave trains in which patterns with differing phase velocities, amplitudes, and wavelengths coexist stably. They are characterized by a larger degree of coherence between the vertical velocity and the thermal and solutal fields and as a result transport more heat and solute than the background state. Consequently, the process of pulse formation can be interpreted as an attempt by the system to maximize heat and solute transport within the constraint imposed by phase conservation, i.e., a fixed number of rolls.

We have focused on the Rayleigh number regime $1.15 R_{R}^{(0)}<R_{T}<1.27 R_{T}^{(0)}$ within which pulse formation is initiated but the phase of the pattern remains well defined. This regime allows one to employ the techniques of phase dynamics, one of the few analytical means available to us for understanding confined states that develop on top of a highly nonlinear state. We have used this approach to gain substantial insight into the properties of these pulses and their multiplicity. These pulses appear to be well described by a thirdorder phase equation. This equation is a particularly simple case of the phase equation describing the evolution of longwavelength Benjamin-Feir instability of traveling waves [20]. To our knowledge this important special case has not hitherto been studied. We have found, using our phase equation, that the pulse shape is given by a third-order nonlinear eigenvalue problem, with the eigenvalue determining the pulse speed. The theory makes predictions about the pulse shape that are consistent with the results of our simulations. These include the fact that pulses traveling more slowly than the group velocity should have oscillatory tails, and that the local wavelength and phase velocity should be linearly related. In addition we verified a general expression relating the pulse speed to the wavelengths and phase velocities within the pulses and without. We interpreted the oscillatory tail of the pulses in terms of a homoclinic orbit to a saddle focus satisfying, under mild assumptions on the phase diffusion and dispersion coefficients, the Shil'nikov condition 0 $<\delta<1$. Using standard theory we established the presence, on the real line, of a great number of coexisting pulse and nonpulse states, and applied this result to establish the existence on a periodic domain of not only single pulses but also of two different types of double (and triple etc.) pulses, all of which move rigidly at constant speed given by the eigenvalue of an appropriate nonlinear eigenvalue problem. These included periodic two-pulse states in which the pulses are identical and their separations equal, and others in which they are not. We exhibited examples of such pulses in the numerical simulations, obtaining them by a process we called "mutation" in which selected parts of the pulse were culled and replicated to create an initial condition of the desired kind. Time integration forward in time was then used to check their stability.
Despite the success of our approach we caution that the phase approach has an important shortcoming. Formally it applies only to states with weak phase gradients. In our pulses wave number changes of order one were common. Thus our states are outside the regime in which the equation should hold. It appears, however, that phase conservation is a sufficiently strong constraint that the phase equation provides a reliable model even outside its formal range of validity.

Another approach to the present problem would be to consider a pulse as the bound state of two fronts, with the pulse width determined by the minimum of the potential between them (if one exists). The small oscillations observed in the widths of the pulses during their propagation (see Sec. IV) could then be interpreted as oscillations about the minimum of this potential. Although such oscillations should decay with time, we have found them to be long lived. Although the most likely explanation of such oscillations (based on their frequency) is that they are an effect of the finite size of the rolls we cannot exclude the possibility that they are the consequence of self-excited oscillations of such bound states (cf. [40]). This approach, which offers scope for a more realistic model of the confined states described here, is planned for a future paper.

The present paper represents the first observation of confined states in thermosolutal convection. These confined states are distinct from the ones found in binary fluid convection. The latter occur very close to onset and consist of localized packets of traveling waves on an otherwise undisturbed background. They also differ physically. In binary fluid mixtures there is no net concentration transport, only concentration migration in response to the applied temperature gradient. Thus the concentration is ultimately pumped horizontally, out of the packet, where it creates regions of negative buoyancy. As a result the packets travel much more slowly than might otherwise be expected. However, because these states are found near onset they are still accessible to description by weakly nonlinear theory [5]. In contrast, in the thermosolutal system solute is transported in the vertical. As a result there is very little tendency towards the kind of concentration braking that is so important in binary fluids. Recent developments in experimental technique [8] suggest that the pulses described here should be amenable to experimental study. The physical considerations just alluded to also suggest that any small-amplitude subcritical localized states present near onset of oscillatory thermosolutal convection should travel faster and have different stability properties than the corresponding ones in binary fluid convection. However, for our parameter values there is an additional difference as well. The subcritical traveling waves present at these parameter values have a second unstable eigenvalue: they are unstable with respect to standing waves, which also bifurcate subcritically. Consequently we do not expect localized packets of traveling waves near onset in our system, although the possibility of small-amplitude confined states of standing waves cannot be excluded.

In their study of localized traveling waves in binary fluids near onset Barten et al. [7] showed that the localized states drifted with a small group velocity in the propagation direction of the phase of its TW components; in the comoving frame these states were found to be time periodic. Different 
localized states differed only in the width of the central part of the pulse while the leading and trailing ends (although different) were found to be the same in all the simulations performed. The velocity, temperature, and concentration profiles could not be described by one common amplitude since their widths and shapes were different. The velocity and temperature amplitudes in the center of the localized states were only slightly smaller than in the extended states at the same values of the parameters. However, the local wavelengths and phase speeds of the rolls increased monotonically as they moved from the trailing toward the leading end. The frequency of the traveling waves in such a pulse was found to be about half the Hopf frequency at the onset, a much larger value than in an extended state, indicating a larger concentration contrast between adjacent rolls and a greater mean lateral concentration flux for the localized states than for the extended ones. The wavelength in the pulse was only about $10 \%$ smaller than in an extended state of similar frequency.

Barten et al. found that the mean buoyancy force in such convection is dominated by the concentration contribution rather than the thermal one. A phase shift between the concentration wave and the velocity wave which takes place in the central part of the localized state drove a mean concentration circulation extending over the whole localized state. This in turn induced a large-scale concentration redistribution. Ahead of the leading front this concentration redistribution produces a barrier that weakens the mean buoyancy force, thereby impeding a rapid invasion of the conductive region by convection. On the other hand, the different widths of the concentration and temperature pulses appear to enhance the buoyancy under both fronts and thereby stabilize the localized state against invasion by the conduction state. The mean concentration flux decreases with decreasing frequency (increasing $R_{T}$ ) and with it the concentration redistribution. This weakens the barrier and increases the forward drift motion of the localized state. Larger frequencies strengthen the barrier and the forward drift velocity of the pulse into the quiescent regime is reduced. These results are in good agreement with the experimental results of Kolodner [41] and with the analytical theory of Riecke [5]. We have identified similar large-scale communication in thermosolutal convection using moving averages. In the reference frame moving with a pulse the small-scale dynamics are fil- tered out, revealing the dynamics on the scale spanning the full horizontal width of the domain and involving large-scale vortices that drift with the pulses. The pulses owe their existence to an interaction between the order one convective scale and this large scale.

We should remark that traveling wave thermosolutal convection has not hitherto been simulated with no-slip boundary conditions, even in domains with basic period of order the roll wavelength. Here, however, our results do not differ markedly from those obtained by Barten et al. for binary mixtures [16]. In particular, the velocity and temperature fields are observed to have similar structure and strength to those in comparable stationary states in pure-fluid convection, with the temperature wave phase shifted slightly relative to the velocity wave, cf. [1]. In contrast to the velocity and temperature components, which at our Rayleigh numbers were nearly harmonic, the solutal component was found to be highly nonharmonic, with an almost trapezoidal profile, indicative of nearly homogeneous solute within each roll, separated by a thin boundary layer that snakes alternately between the top and bottom of the layer. This is a consequence of the "mirror-glide" symmetry which in turn is a consequence of imposing identical boundary conditions at the top and bottom of the layer. The lateral mean flow in a uniform wave train was found to be several orders of magnitude smaller than the phase velocity of the wave. A small but significant mean lateral convective heat flux, ranging between $0.006 N_{T}$ and $0.014 N_{T}$, is generated by the phase shift between the vertical velocity of the fluid and the temperature wave, with a similar solute flux, between $0.015 \mathrm{~N}_{S}$ and $0.038 N_{S}$, generated by the phase difference between the convection and the solutal wave. Such mean flows and fluxes were also found in simulations of binary fluid mixtures [16].

\section{ACKNOWLEDGMENTS}

This work was supported in part by the National Science Foundation under Grant Nos. DMS-9406144 and ECS9217394, and by the National Aeronautics and Space Administration under Grant No. NAG5-2256. E.K. thanks JILA, University of Colorado, Boulder, Colorado, for financial support. We are grateful to Keith Julien for assistance with the NRK package, and to Michael Proctor for helpful comments.
[1] E. Knobloch, A. E. Deane, J. Toomre, and D. R. Moore, Contemp. Math. 56, 203 (1986); A. E. Deane, E. Knobloch, and J. Toomre, Phys. Rev. A 36, 2862 (1987).

[2] E. Knobloch, A. E. Deane, and J. Toomre, Contemp. Math. 99. 339 (1989)

[3] In annular geometry such states were first reported by J. J. Niemela, G. Ahlers, and D. S. Cannell, Phys. Rev. Lett. 64, 1365 (1990); see also K. A. Anderson and R. P. Behringer, Phys. Lett. A 145, 323 (1990); and P. Kolodner and J. A. Glazier, Phys. Rev. A 42, 7504 (1990).

[4] O. Thual and S. Fauve, J. Phys. (Paris) 49, 1829 (1988).

[5] H. Riecke, Phys. Rev. Lett. 68, 301 ( 1992); Physica D 61, 253 (1992).

[6] N. Balmforth, Annu. Rev. Fluid Mech. 27, 335 (1995).
[7] W. Barten, M. Lücke, M. Kamps, and R. Schmitz, Phys. Rev. E 51, 5662 (1995).

[8] A. A. Predtechensky, W. D. McCormick, J. B. Swift, Z. Noszticzius, and H. L. Swinney, Phys. Rev. Lett. 72, 218 (1994); A. A. Predtechensky, W. D. McCormick, J. B. Swift, A. G. Rossberg, and H. L. Swinney, Phys. Fluids 6. 3923 (1994).

[9] E. Knobloch, Phys. Rev. A 34, 1538 (1986).

[10] Y. Y. Renardy, Phys. Fluids A 5, 1376 (1993); and private communication.

[11] E. Knobloch, Contemp. Math. 56, 193 (1986).

[12] P. M. Gresho, Annu. Rev. Fluid Mech. 23, 413 (1991).

[13] L. Quartapelle, Numerical Solution of the Incompressible Navier-Stokes Equations (Birkhäuser, Basel, 1993).

[14] A. E. Deane, Ph.D. thesis, University of Colorado, 1987. 
[15] W. Barten, M. Lücke, W. Hort, and M. Kamps, Phys. Rev. Lett. 63, 376 (1989).

[16] W. Barten, M. Lücke, M. Kamps, and R. Schmitz, Phys. Rev. E 51, 5636 (1995).

[17] B. J. Gluckman, P. Marcq, J. Bridger, and J. P. Gollub, Phys. Rev. Lett. 71, 2034 (1993).

[18] E. Knobloch and J. B. Weiss, Phys. Rev. A 36, 1522 (1987).

[19] G. B. Whitham, Linear and Nonlinear Waves (Wiley, New York, 1974).

[20] B. Janiaud, A. Pumir, D. Bensimon, V. Croquette, H. Richter, and L. Kramer, Physica D 55, 269 (1992).

[21] H. Chaté and P. Manneville, in Turbulence: A Tentative Dictionary, edited by P. Tabeling and O. Cardoso (Plenum, New York, 1994).

[22] H. R. Brand and R. J. Deissler, Phys. Rev. A 41, 5478 (1990).

[23] R. J. Deissler, Y. C. Lee, and H. R. Brand, Phys. Rev. A 42 , 2101 (1990).

[24] Y. Kuramoto, Prog. Theor. Phys. 71, 1182 (1984).

[25] C. J. Chapman and M. R. E. Proctor, J. Fluid Mech. 101, 759 (1980).

[26] P. Kolodner, Phys. Rev. Lett. 66, 1165 (1991). This paper demonstrates that the supercritical confined states found in Ref. [3] do travel in a sufficiently uniform experimental cell.

[27] W. Schöpf and L. Kramer, Phys. Rev. Lett. 66, 2316 (1991).

[28] A. Améodo, P. Coullet, E. Spiegel, and C. Tresser, Physica D 14, $327(1985)$.
[29] Y. Liu and R. E. Ecke (unpublished).

[30] L. P. Shil'nikov, Sov. Math. Dokl. 6, 163 (1965); for a study of Shil'nikov dynamics of standing waves in thermosolutal convection see E. Knobloch, D. R. Moore, J. Toomre, and N. O. Weiss, J. Fluid Mech. 166, 409 (1986).

[31] P. Glendinning and C. Sparrow, J. Stat. Phys. 35, 645 (1984).

[32] P. Gaspard, Phys. Lett. 97A, 1 (1983); for related earlier work see J. W. Evans, N. Fenichel, and J. A. Feroe, SIAM (Soc. Ind. Appl. Math.) J. Appl. Math. 42, 219 (1982).

[33] S. Wiggins, Global Bifurcations and Chaos (Springer, New York, 1988).

[34] S. V. Gonchenko, D. V. Turaev, P. Gaspard, and G. Nicolis, Nonlinearity 10, 409 (1997).

[35] Y.-T. Lau, Int. J. Bifurcation Chaos 2, 543 (1992).

[36] P. Henrici, Discrete Variable Methods in Ordinary Differential Equations (Wiley, New York 1962); J. R. Cash and D. R. Moore, B.I.T. 20, 44 (1980).

[37] J. W. Evans, Indiana U. Math. J. 22, 577 (1972); 25, 301 (1976). See Ref. [6] for additional references.

[38] C. Elphick, E. Meron, J. Rinzel, and E. A. Spiegel, J. Theor. Biol. 146, 249 (1990).

[39] C. Elphick, E. Meron, and E. A. Spiegel, SIAM (Soc. Ind. Appl. Math.) J. Appl. Math. 50, 490 (1990).

[40] R. Deissler and H. R. Brand, Phys. Rev. Lett. 72, 478 (1994).

[41] P. Kolodner, Phys. Rev. A 44, 6448 (1991); Phys. Rev. E 50, 2371 (1994). 

